NATIONAL LABORATORY

\title{
Real-Time Dynamic Brake Assessment Proof of Concept Test Final Report
}

\section{November 2011}

Prepared by

Mary Beth Lascurain Hudson

Oscar Franzese

Gary Capps

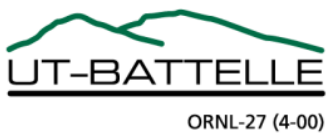




\section{DOCUMENT AVAILABILITY}

Reports produced after January 1, 1996, are generally available free via the U.S. Department of Energy (DOE) Information Bridge.

Web site http://www.osti.gov/bridge

Reports produced before January 1, 1996, may be purchased by members of the public from the following source.

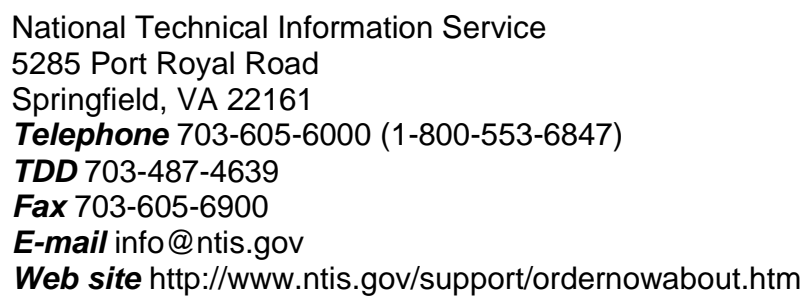

Reports are available to DOE employees, DOE contractors, Energy Technology Data Exchange (ETDE) representatives, and International Nuclear Information System (INIS) representatives from the following source.

Office of Scientific and Technical Information

P.O. Box 62

Oak Ridge, TN 37831

Telephone 865-576-8401

Fax 865-576-5728

E-mail reports@osti.gov

Web site http://www.osti.gov/contact.html

This report was prepared as an account of work sponsored by an agency of the United States Government. Neither the United States Government nor any agency thereof, nor any of their employees, makes any warranty, express or implied, or assumes any legal liability or responsibility for the accuracy, completeness, or usefulness of any information, apparatus, product, or process disclosed, or represents that its use would not infringe privately owned rights. Reference herein to any specific commercial product, process, or service by trade name, trademark, manufacturer, or otherwise, does not necessarily constitute or imply its endorsement, recommendation, or favoring by the United States Government or any agency thereof. The views and opinions of authors expressed herein do not necessarily state or reflect those of the United States Government or any agency thereof. 
Energy and Transportation Science Division

\title{
REAL-TIME DYNAMIC BRAKE ASSESSMENT PROOF OF CONCEPT TEST FINAL REPORT
}

\author{
Mary Beth Lascurain Hudson \\ Oscar Franzese \\ Gary Capps
}

Date Published: November 2011

Prepared by

OAK RIDGE NATIONAL LABORATORY

Oak Ridge, Tennessee 37831-6283

managed by

UT-BATTELLE, LLC

for the

U.S. DEPARTMENT OF ENERGY

under contract DE-AC05-00OR22725 



\section{CONTENTS}

Page

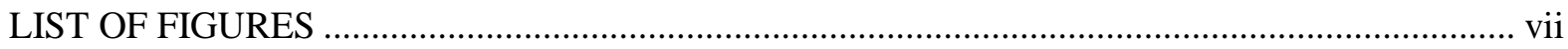

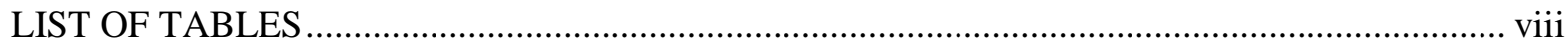

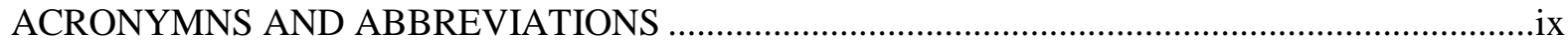

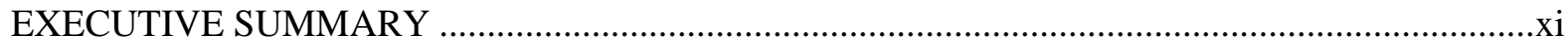

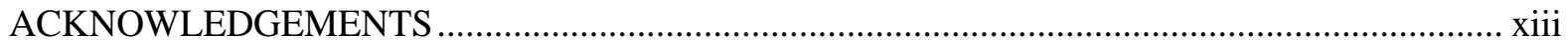

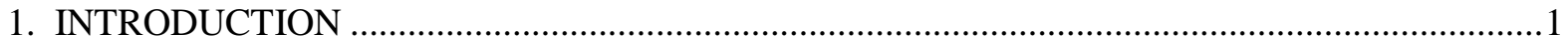

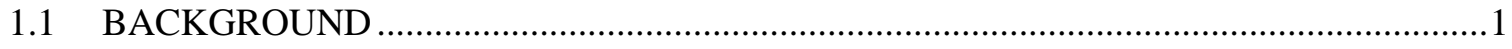

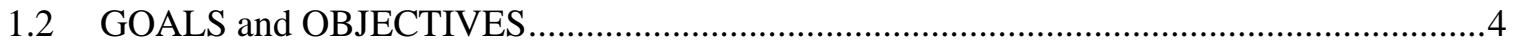

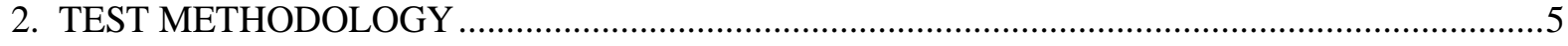

2.1 REAL-TIME BRAKE ASSESSMENT THEORY ….....................................................

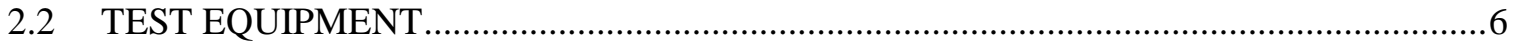

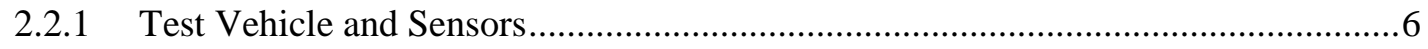

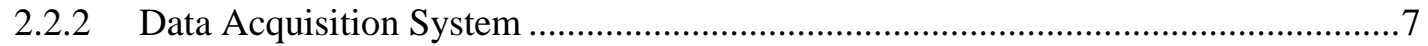

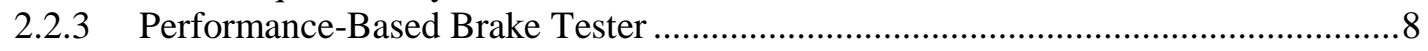

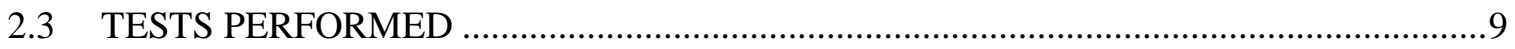

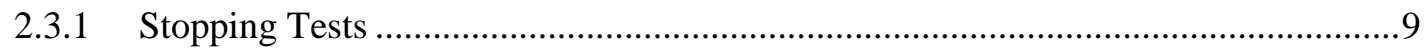

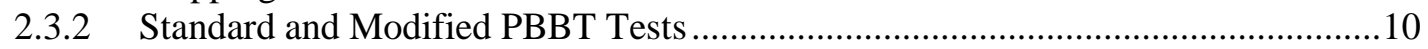

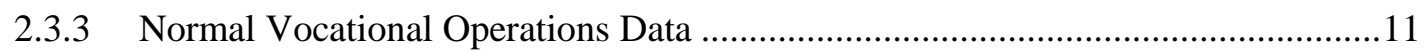

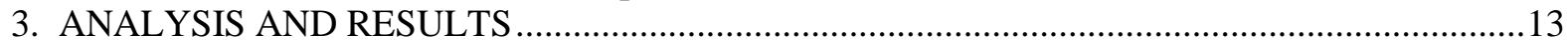

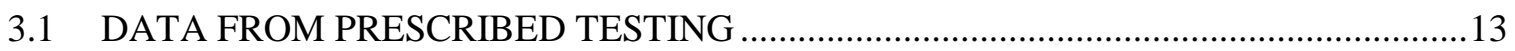

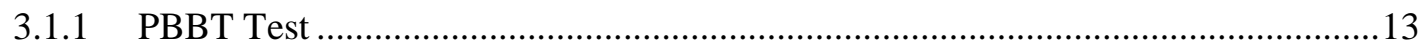

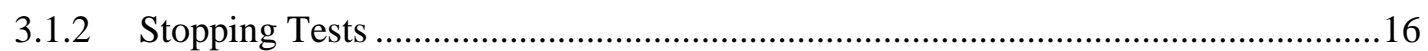

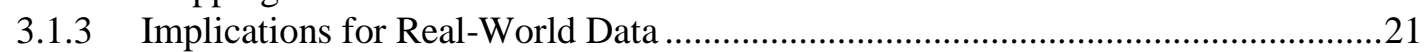

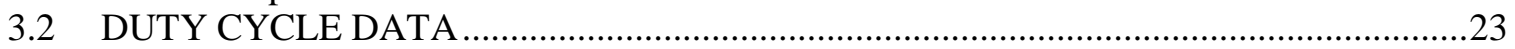

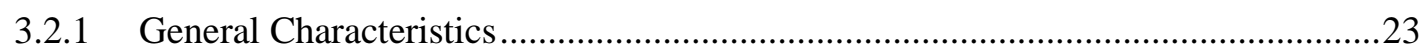

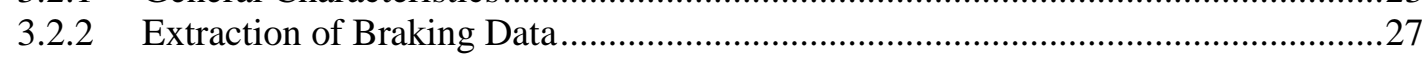

3.3 COMPARISON OF REAL-WORLD AND SCRIPTED STOPPING DATA ......................31

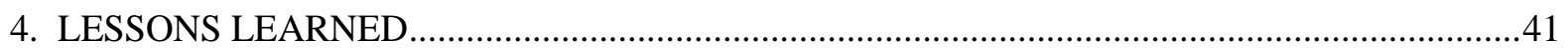

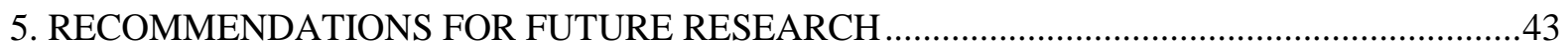

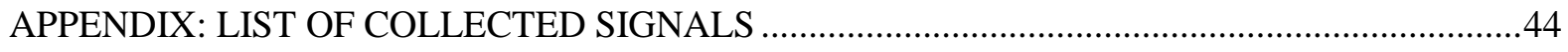




\section{LIST OF FIGURES}

\section{Page}

Figure 1. Comparison of 20-mph stopping distance and brake efficiency..........................................

Figure 2. Plot of actual vs. predicted stopping test from simplified model. ........................................2

Figure 3. Braking force vs. brake application pressure for several stopping tests.............................. 3

Figure 4. Total brake force vs brake application pressure from several PBBT tests (individual test

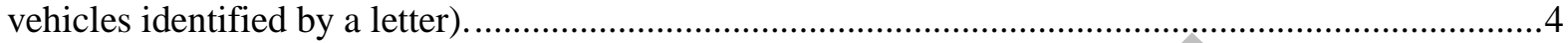

Figure 5. Distribution of brake application pressure in MTDC combination vehicle data...................5

Figure 6. Theoretical plot of how real-world data might be able to predict PBBT information............6

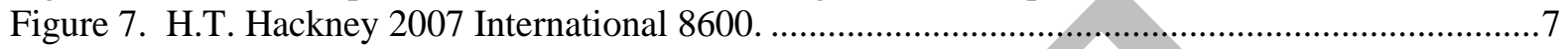

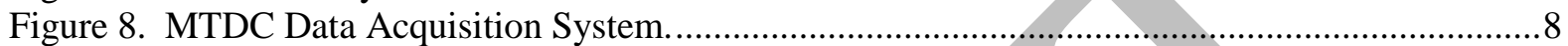

Figure 9. Performance-based brake tester located at the Greene County Inspection Station (roller-

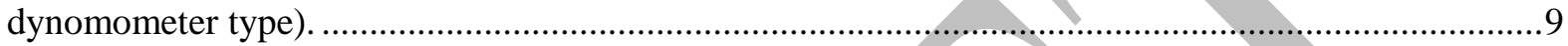

Figure 10. Conceptual trace of brake application pressure for regular PBBT test.............................10

Figure 11. Conceptual trace of brake application pressure for stepped-pressure PBBT test...............11

Figure 12. Data from stepped-pressure PBBT test...................................................................13

Figure 13. Brake application pressure and brake force extracted from stepped-pressure PBBT test. .14

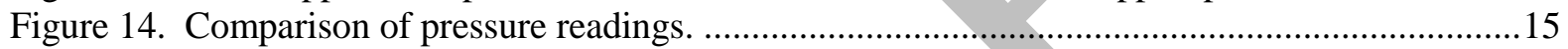

Figure 15. Comparison of stepped-pressure PBBT results for two loading conditions......................16

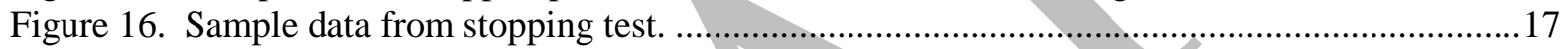

Figure 17. Effect of starting speed on deceleration-pressure profile. ............................................. 18

Figure 18. Summary plot of medium-load test data...................................................................... 19

Figure 19. Summary plot of heavy-load test data. .....................................................................20

Figure 20. Conceptual plot of effect of loading conditions on pressure-deceleration relationship......22

Figure 21. Conceptual plot of evidence of braking system problems on pressure-deceleration plot...23

Figure 22. Speed distribution of regular duty cycle data.............................................................24

Figure 23. Distribution of brake application pressures (greater than zero) in duty cycle data. ...........25

Figure 24. Distribution of steer axle weight in duty cycle data. ..................................................26

Figure 25. Distribution of drive axle weight in regular duty cycle data. .......................................26

Figure 26. Bivariate speed and pressure distribution in regular duty cycle data. ..............................27

Figure 27. Brake application pressure and vehicle speed vs. time (test data segment)......................28

Figure 28. Altitude and vehicle speed vs. distance traveled (test data segment)..............................29

Figure 29. Brake application pressure and vehicle speed vs. time (field data segment).....................30

Figure 30. Altitude and vehicle speed vs. distance traveled (field data segment).............................31

Figure 31. Deceleration vs. brake application pressure - test and field data - medium load................32

Figure 32. Vehicle weight (from on-board sensor) vs. brake application pressure - test data -

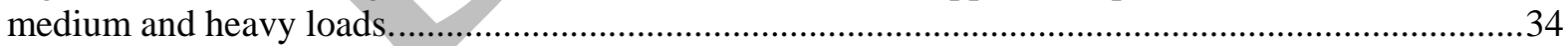

Figure 33. Sensor weight vs. adjusted weight - field data - medium load. .......................................35

Figure 34. Deceleration vs. brake application pressure - test and field data with weight adjustment -

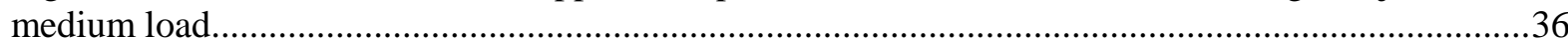

Figure 35. Deceleration vs. brake application pressure - test and field data with weight adjustment:

no outliers - medium load. 


\section{LIST OF TABLES}

Page

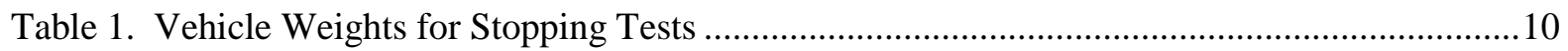

Table 2. Test Statistics - Medium Load .........................................................................................29

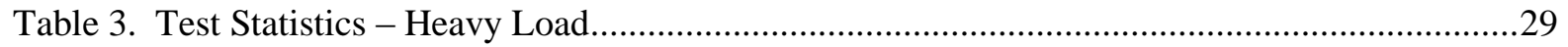

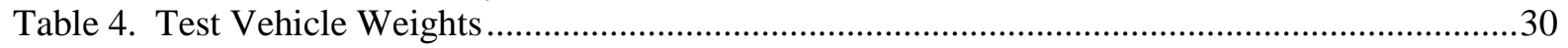

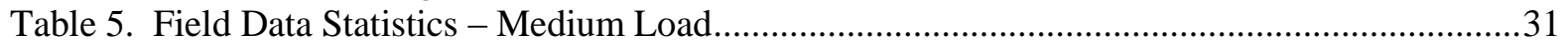

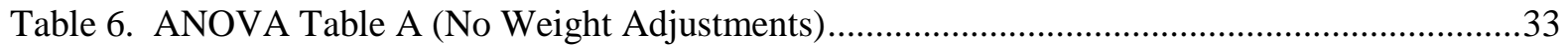

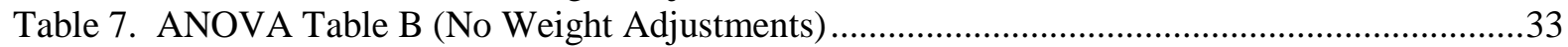

Table 8. Field Data Statistics -Medium Load - Original and Adjusted Weight .................................36

Table 9. Field Data Statistics - Medium Load - Adjusted Weight .....................................................37

Table 10. Field Data Statistics - Medium Load - Adjusted Weight - No Outliers .............................37

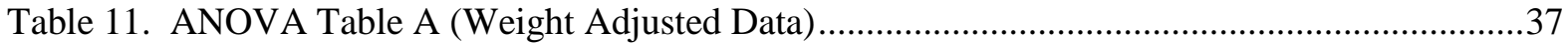

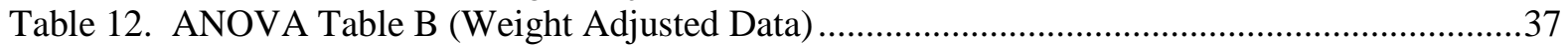




\section{ACRONYMNS AND ABBREVIATIONS}

$\begin{array}{ll}\text { Term } & \text { Definition } \\ \text { ANOVA } & \text { Analysis of Variance } \\ \text { BAPr } & \text { Brake Application Pressure } \\ \text { BE } & \text { Brake Efficiency } \\ \text { BF } & \text { Brake Force } \\ \text { DAS } & \text { Data Acquisition System } \\ \text { Dec } & \text { Deceleration } \\ \text { ft } & \text { Feet } \\ \text { g } & \text { Gravitational acceleration(s) } \\ \text { GPS } & \text { Global Positioning System } \\ \text { GVW } & \text { Gross Vehicle Weight } \\ \text { Hz } & \text { Hertz } \\ \text { lbs } & \text { Pounds } \\ \text { mph } & \text { Miles per hour } \\ \text { MTDC } & \text { Medium Truck Duty Cycle } \\ \text { P } & \text { Pressure } \\ \text { P-BF } & \text { Pressure vs. Brake Force } \\ \text { PBBT } & \text { Performance-Based Brake Tester } \\ \text { psi } & \text { Pounds per square inch } \\ \text { s } & \text { Seconds } \\ \text { SDw } & \text { Steer Drive Weight }\end{array}$




\section{EXECUTIVE SUMMARY}

This proof-of-concept research was performed to explore the feasibility of using real-world braking data from commercial motor vehicles to make a diagnosis of brake condition similar to that of the performance-based brake tester (PBBT). This was done by determining the relationship between pressure and brake force (P-BF), compensating for the gross vehicle weight (GVW). The nature of this P-BF relationship (e.g., low braking force for a given brake application pressure) may indicate brake system problems.

In order to determine the relationship between brake force and brake application pressure, a few key parameters of duty cycle information were collected. Because braking events are often brief, spanning only a few seconds, a sample rate of $10 \mathrm{~Hz}$ was needed. The algorithm under development required brake application pressure and speed (from which deceleration was calculated). Accurate weight estimation was also needed to properly derive the braking force from the deceleration. In order to ensure that braking force was the predominant factor in deceleration for the segments of data used in analysis, the data was screened for grade as well. Also, the analysis needed to be based on pressures above the crack pressure. The crack pressure is the pressure below which the individual brakes are not applied due the nature of the mechanical system. This value, which may vary somewhat from one wheel end to another, is approximately $10 \mathrm{psi}$. Therefore, only pressures $15 \mathrm{psi}$ and above were used in the analysis. The Department of Energy's Medium Truck Duty Cycle research has indicated that under the real-world circumstances of the test vehicle brake pressures of up to approximately 30 psi can be expected.

Several different types of data were collected during the testing task of this project. Constant-pressure stopping tests were conducted at several combinations of brake application pressure $(15,20,25$, and 30 psi), load conditions (moderately and fully laden), and speeds (20 and $30 \mathrm{mph}$ ). Data was collected at $10 \mathrm{~Hz}$. Standard and stepped-pressure performance-based brake tests with brake pressure transducers were performed for each loading condition. The stepped-pressure test included the constant-pressure intervals of brake application at $15,20,25$, and 30 psi. The PBBT data files included $10 \mathrm{~Hz}$ streaming data collected during the testing of each axle. Two weeks of real-world duty cycle (driving and braking) data was also collected at $10 \mathrm{~Hz}$.

Initial analysis of the data revealed that the data collected in the field (i.e., day-to-day operations) provided the same information as that obtained from the controlled tests. Analysis of the data collected revealed a strong linear relationship between brake application pressure and deceleration for given GVWs. As anticipated, initial speed was not found to be a significant factor in the deceleration-pressure relationship, unlike GVW.

The positive results obtained from this proof of concept test point to the need for further research to expand this concept. A second phase should include testing over a wider range of speeds and include medium brake application pressures in addition to the low pressures tested in this research. Testing on multiple vehicles would also be of value. This future phase should involve testing to determine how degradation of braking performance affects the pressure-deceleration relationship. 


\section{ACKNOWLEDGEMENTS}

The authors would like to thank the H.T. Hackney Company for providing the test vehicle, fuel, and driver for this research project. We would especially like to thank their operations manager, Mike Moore and shop manager, Calvin Patterson, for making the scripted testing possible.

We would also like to thank MGM Brakes, Inc., a division of Indianhead Industries, for customizing their brake monitoring system for this research.

We would like to thank the Tennessee Department of Safety for making the Greene County Inspection Station facility available for testing. 


\section{INTRODUCTION}

North American Standard Level-1commercial motor vehicle inspection includes a component to visually inspect the vehicle's braking system. Because it is a visual method, the traditional Level-1 inspection does not look at actual brake performance. The performance-based brake tester (PBBT) provides a quantitative indicator of vehicle braking ability. This research seeks to explore, through proof-of-concept testing, the feasibility of developing an on on-board system to provide a similar real-time brake indicator based on dynamic stopping test data collected on-board the vehicle.

\subsection{BACKGROUND}

Previous research has confirmed that although the PBBT brake efficiency (BE) may be considered in terms of deceleration, it is not a clear indicator of the actual distance required to execute a panic stop. This is evident from 20-mph stopping test data collected Figure 1. The data for this background research was collected by Battelle Memorial Institute (Battelle) at the Greene County Inspection Station in years 2008-2009.

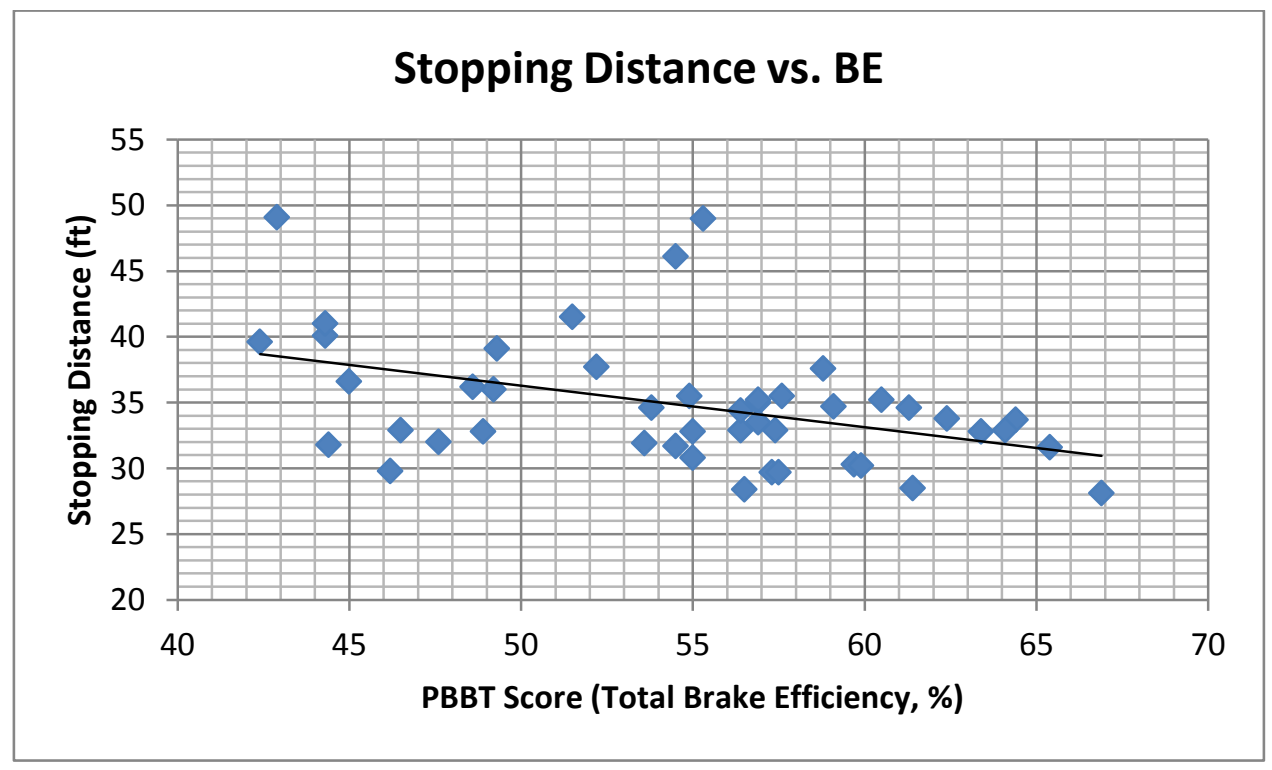

Figure 1. Comparison of 20-mph stopping distance and brake efficiency.

The lack of a clear relationship between stopping distance and BE as seen in Figure 1 prompted further investigation into the fundamental relationships between stopping distance and BE in order to determine possible causes for discrepancy.

Follow-on research involved efforts to use the PBBT test results to predict stopping distance (i.e., develop an algorithm for a synthetic stopping distance test). The data for this effort came from the Battelle stopping distance tests. The algorithm was based on the idea that deceleration determines stopping distance. This deceleration is a function of the applied forces on the vehicle; during a stopping event, these forces come primarily from the braking force (BF) generated by the vehicle's braking system. The magnitude of this BF is dependent (although not solely) on the brake application pressure (P). When the pressure transducer is used, the PBBT test provides the relationship between the brake application pressure and the BF. Estimating stopping distances from the P-BF relationship given by the PBBT yielded results better than the simple linear model relating $\mathrm{BE}$ and stopping distance, but the correlation 
was weak, indicating that at least some of the assumptions made in the development of the algorithm were incorrect (Figure 2).

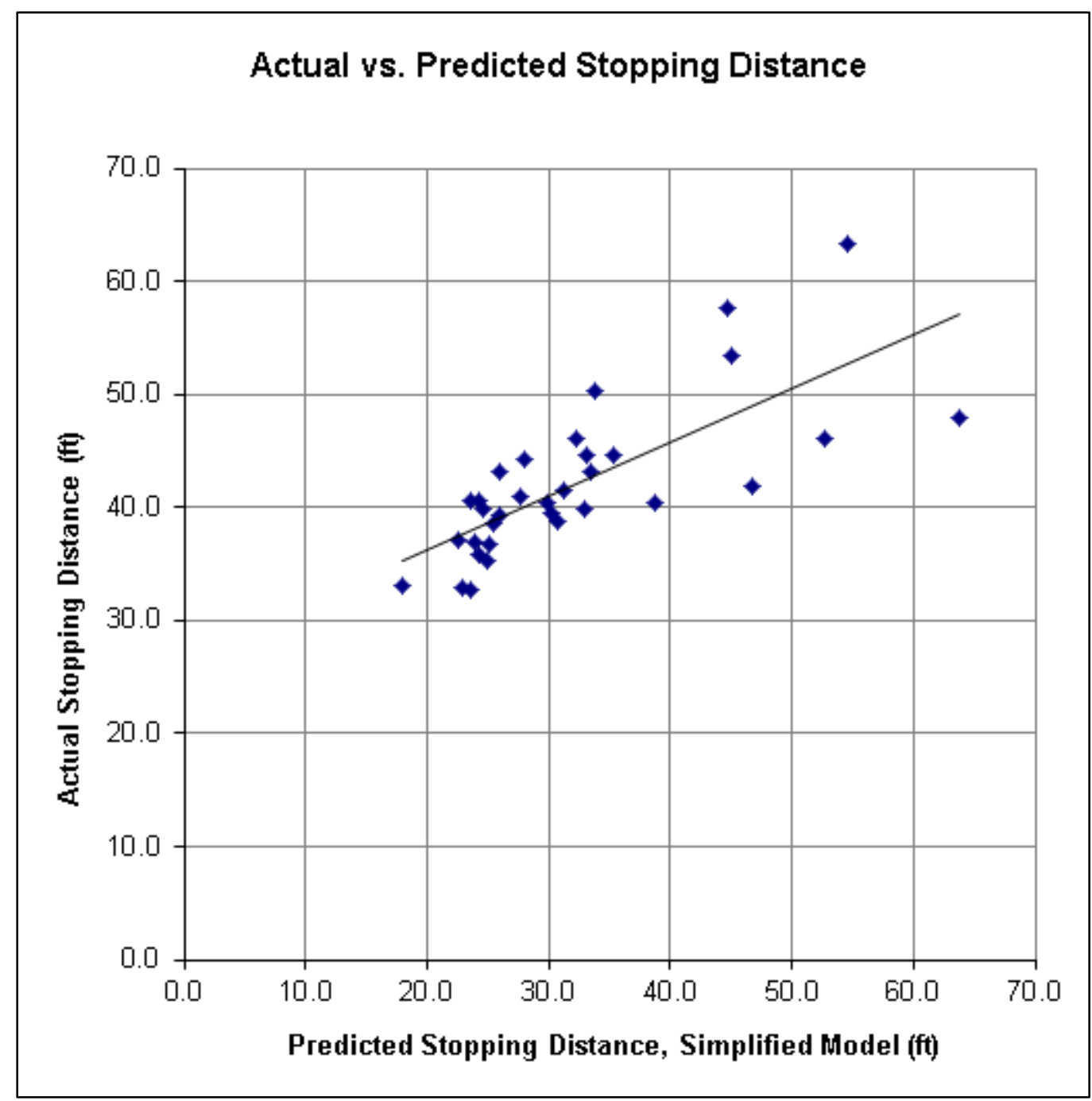

Figure 2. Plot of actual vs. predicted stopping test from simplified model.

Upon further investigation, it was discovered that the P-BF relationship, which appeared to be linear in the pressure region tested in a typical PBBT (up to approximately $60 \mathrm{psi}$ ), is nonlinear in the higher pressures where the panic stops are performed in stopping distance tests (up to approximately $100 \mathrm{psi}$ ). Data from several 20-mph stopping distance tests from a single vehicle is shown in Figure 3. 


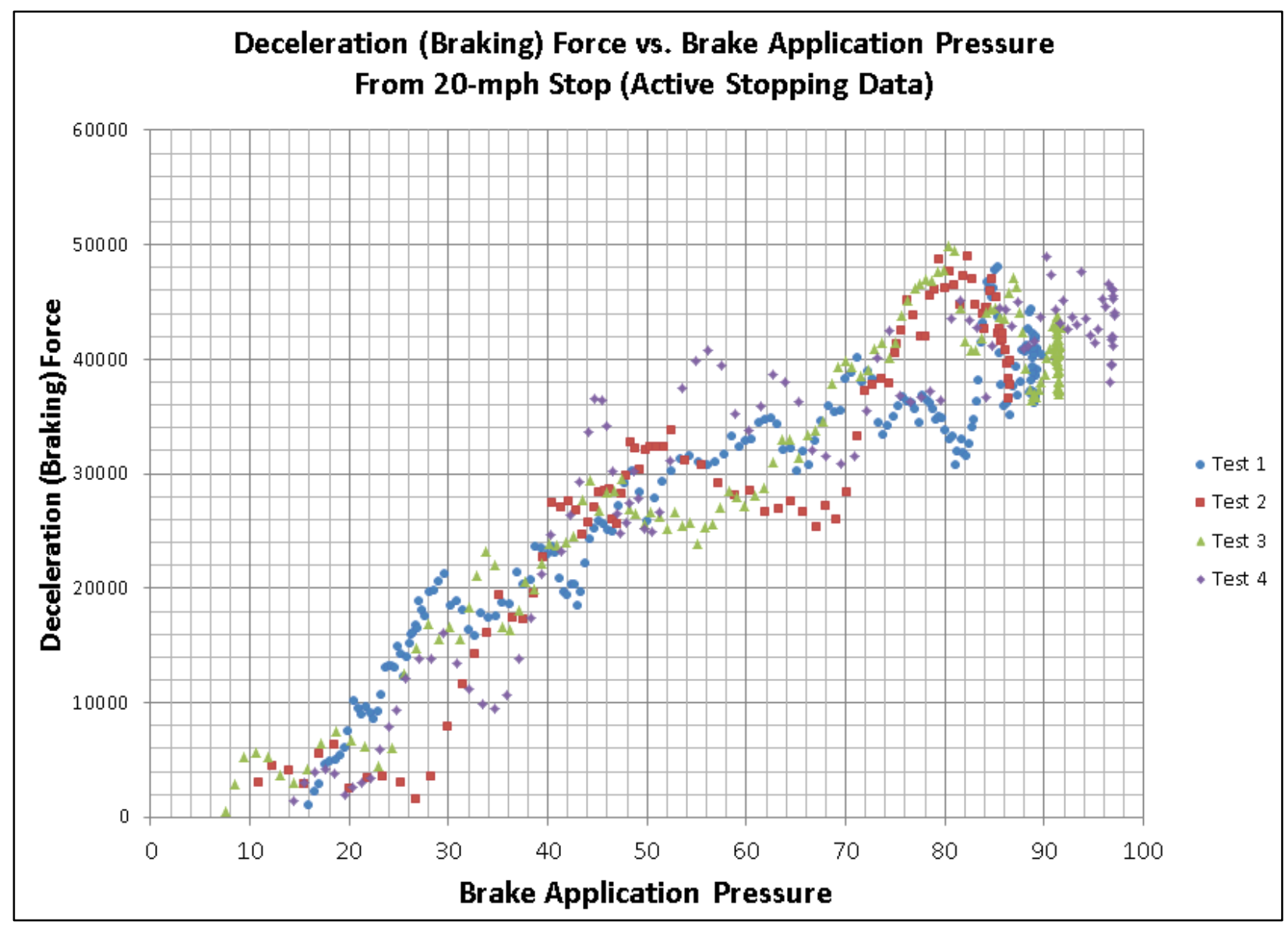

Figure 3. Braking force vs. brake application pressure for several stopping tests.

Although the relationship between force and pressure is highly nonlinear across the full range of brake application pressure, it is piecewise linear in the lower-to-mid pressure ranges. This pressure range (below 40-50 psi) is in the range of the typical PBBT test as shown in Figure 4. 


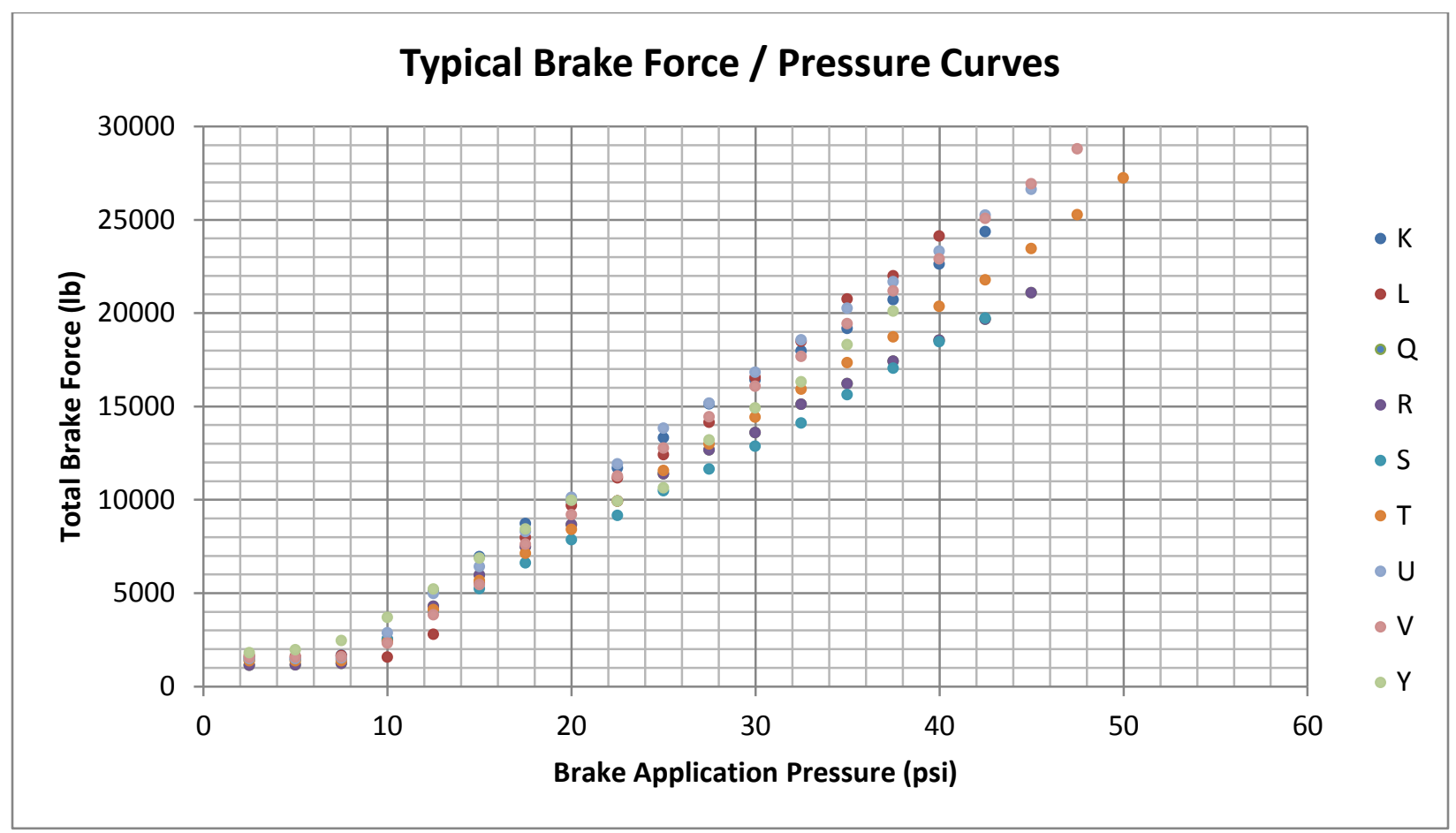

Figure 4. Total brake force vs brake application pressure from several PBBT tests (individual test vehicles identified by a letter).

Unlike previous research conducted with the idea of determining stopping distance from the PBBT results, this research builds on previous work to use real-world stopping data to predict PBBT score or make a similar diagnosis of brake condition based on performance. For example, changes in the P-BF relationship over the life of the brakes may indicate brake system problems. Further research may indicate a minimum "standard" for the P-BF relationship corresponding to properly functioning brakes (i.e., a line on the P-BF graph that the brakes should perform above).

\subsection{GOALS AND OBJECTIVES}

The goal of this research was to determine the feasibility of developing an on-board brake assessment system based on real-time dynamic data collected from real-world braking events. The objective for this proof-of concept phase was to determine whether a strong relationship could be found between BF (measured by deceleration and weight) and brake application pressure from real-world data. Several controlled tests were performed involving both the PBBT and scripted stopping tests to determine such a relationship. Equivalent real-world data was then analyzed to determine whether a similar force-pressure relationship could be obtained from normal operational data. Such a relationship could be used in a realworld environment to provide a real-time safety indicator of brake performance. 


\section{TEST METHODOLOGY}

\subsection{REAL-TIME BRAKE ASSESSMENT THEORY}

A distribution of real-world braking data collected in partnership with the Department of Energy's Medium Truck Duty Cycle (MTDC) effort (a year of vehicle data from three combination vehicles) indicates that virtually all brake applications occur at pressures below $30 \mathrm{psi}$, as shown in Figure 5. This means that most braking events taking place during the normal duty cycle of the vehicles studied tend to occur at pressures much lower than those reached in either the 20-mph panic stops ( $90 \mathrm{psi}$ ) or performance-based brake tests ( $60 \mathrm{psi})$.

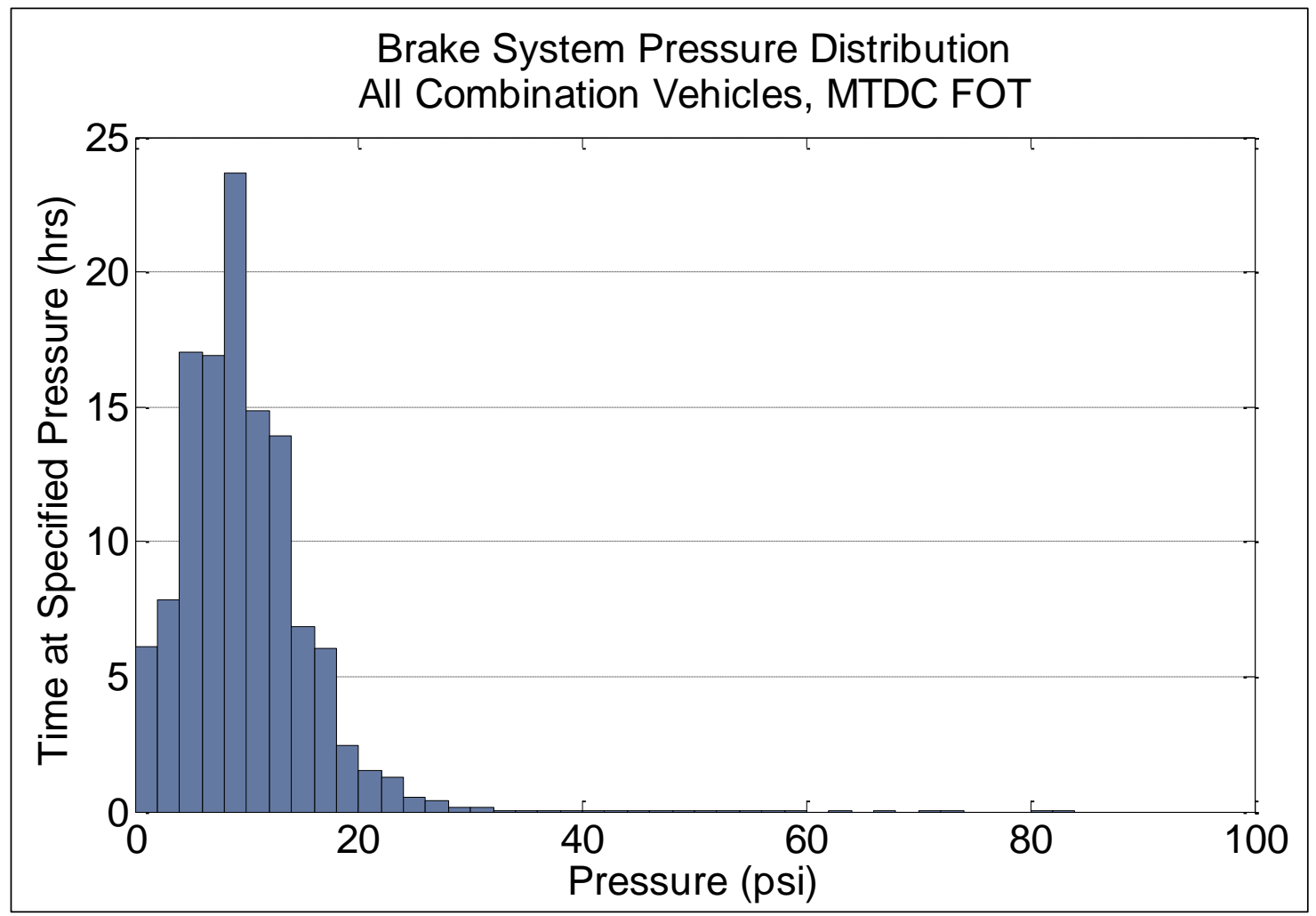

Figure 5. Distribution of brake application pressure in MTDC combination vehicle data.

Due to the nonlinear relationship between the brake application pressure and corresponding force (directly proportional to deceleration), the low typical application pressures present a problem for predicting brake performance in a panic stop based on PBBT or real-world data. However, the piecewise linearity of the pressure-force curve in the lower-pressure region may make it possible to extrapolate braking forces in this linear region for pressures higher than those seen in typical real-world braking data. This concept is illustrated in Figure 6. 


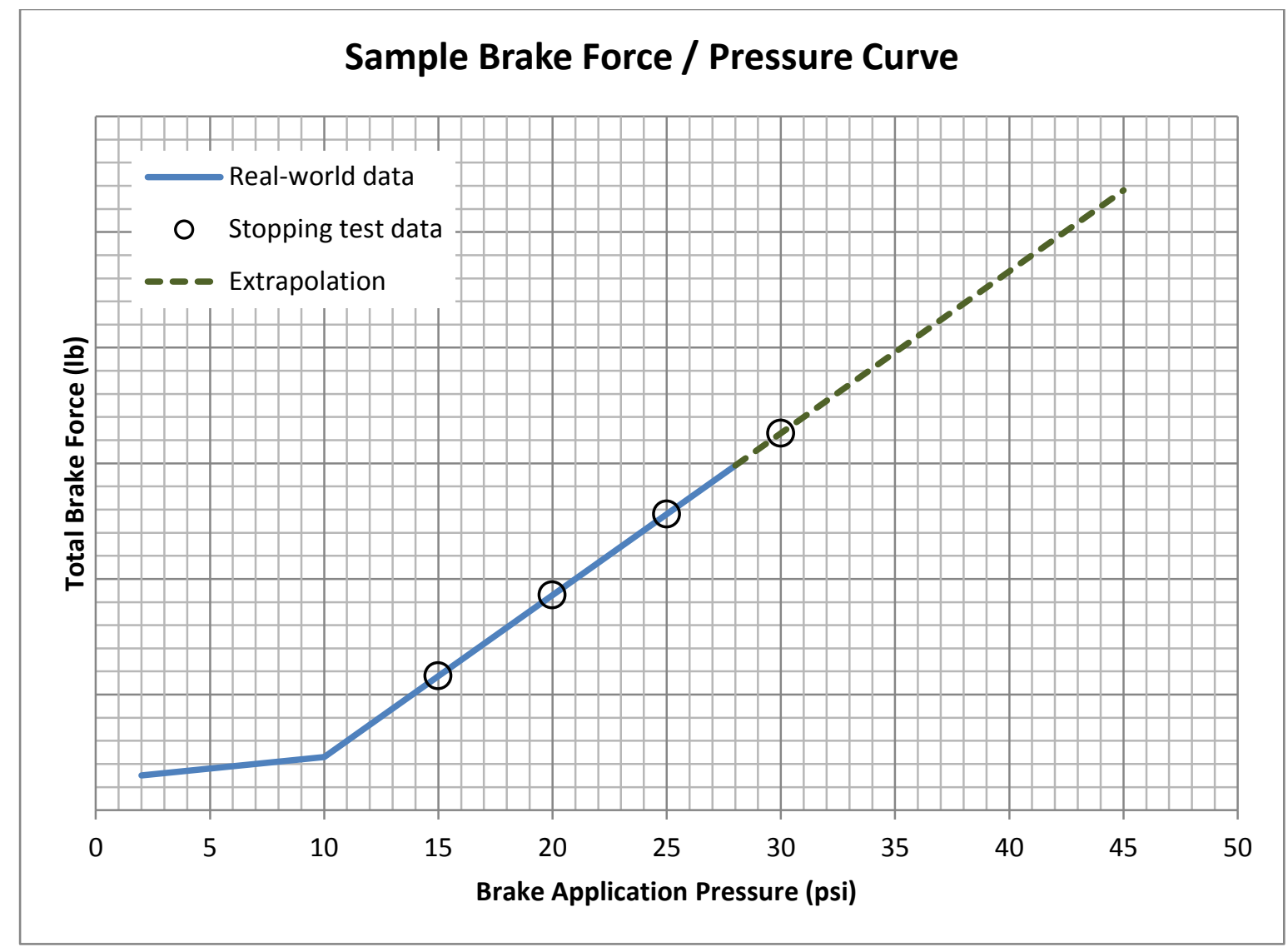

Figure 6. Theoretical plot of how real-world data might be able to predict PBBT information.

In order to determine the relationship between BF and brake application pressure, a few key parameters of duty cycle information needed to be collected. Because braking events are often brief, spanning only a few seconds, a sample rate of $10 \mathrm{~Hz}$ was needed. The algorithm to be developed requires brake application pressure and speed (from which deceleration is calculated). Accurate weight estimation was also needed to properly derive the braking force from the deceleration. In order to ensure that braking forces is the predominant factor in deceleration for the segments of data used in analysis, it was determined that the data should be screened for grade as well. Also, the analysis needed to be based on pressures above the crack pressure. The individual brakes will not be applied below this pressure of approximately 10 psi. Therefore, only pressures of $15 \mathrm{psi}$ and above were used in the analysis. The MTDC research indicated that under the real-world circumstances of the test vehicle, brake pressures of up to approximately 30 psi can be expected; thus, this was the highest pressure tested in this effort.

\subsection{TEST EQUIPMENT}

\subsubsection{Test Vehicle and Sensors}

One of the H.T. Hackney combination test vehicles from the MTDC project was used for this project (Figure 7). 


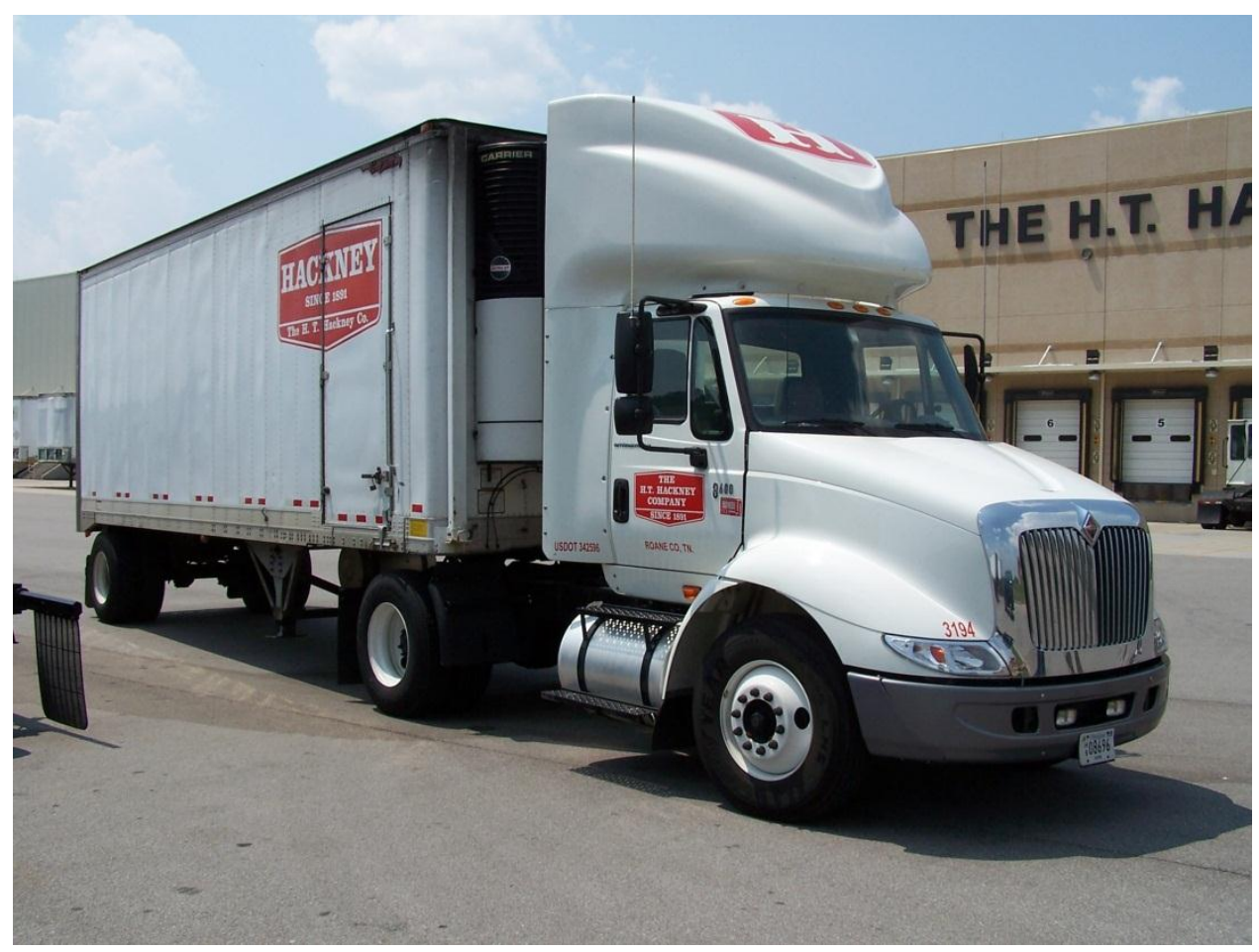

Figure 7. H.T. Hackney 2007 International 8600.

This vehicle is a Class-7 combination tractor-trailer used in local delivery to convenience stores. It is a 2007 International 8600 day-cab tractor which regularly hauls 28 -ft pup trailers. The test vehicle had all cabling and sensors already installed from the previous MTDC research effort.

\subsubsection{Data Acquisition System}

A data acquisition system (DAS) was placed on each test vehicle to collect relevant duty cycle data for wireless upload and subsequent analysis. Native signals were obtained directly from the vehicle's J1939 and J1708 data buses. The DAS enclosure is shown in Figure 8. 


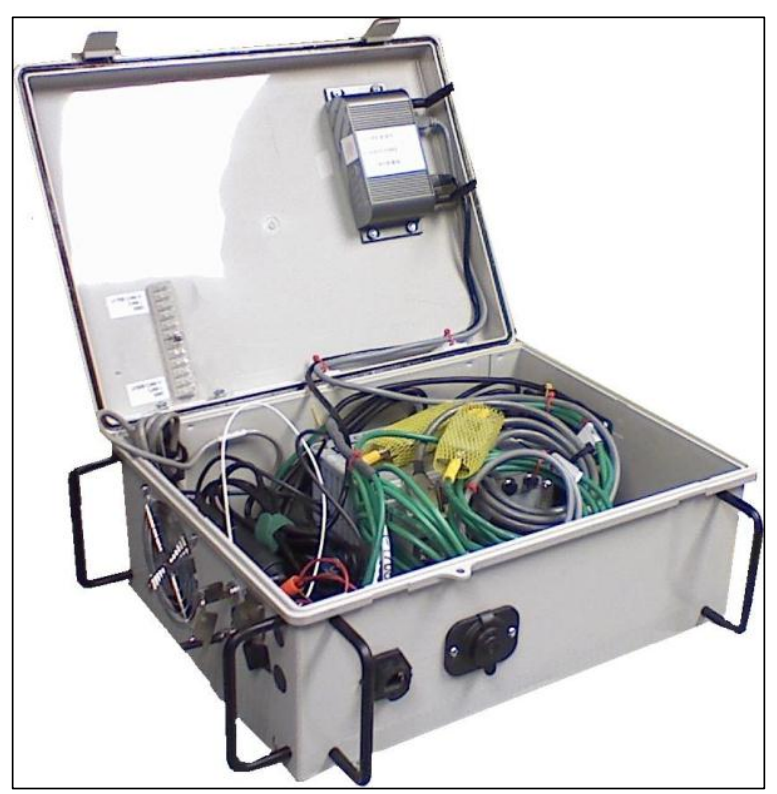

Figure 8. MTDC Data Acquisition System.

The VBOX II Lite collected Global Positioning System (GPS)-related information including speed, acceleration, and GPS at a rate of $5 \mathrm{~Hz}$. This data was communicated via the CAN (J1939) protocol. The eDAQ-lite data acquisition unit collected and stored data from the vehicle's J1939 and J1708 data bus and connected J1939 sensors. For the purposes of the field operational test, it was configured to record all data at a rate of $10 \mathrm{~Hz}$. The eDAQ-lite was the central data collection unit with internal data storage for use between periodic uploads. A display was used to provide speed and brake application pressure information to the driver for the scripted stopping tests.

The Air-Weigh LoadMaxx is a self-weighing system which determines the vehicle's gross weight by means of pressure transducers and posts the weight to the vehicle's J1939 data bus. The Air-Weigh system provided real-time tractor axle group weight information. The MGM e-Stroke on-board brake monitoring system included a transducer to measure brake application pressure. This sensor was modified to provide this parameter at a refresh rate of $10 \mathrm{~Hz}$, well above the usual $1-\mathrm{Hz}$ setting. Other parameters of potential interest were obtained from the J1939 and J1708 data buses native to the vehicle (see Appendix).

\subsubsection{Performance-Based Brake Tester}

PBBTs are devices that can evaluate the current BE of a vehicle through the measurement of brake forces developed as a vehicle engages in a braking event while on a PBBT (Figure 9). PBBT devices are typically in-ground, but can also be in portable configurations. The common types of PBBTs include roller dynamometers and flat-plate testers. 


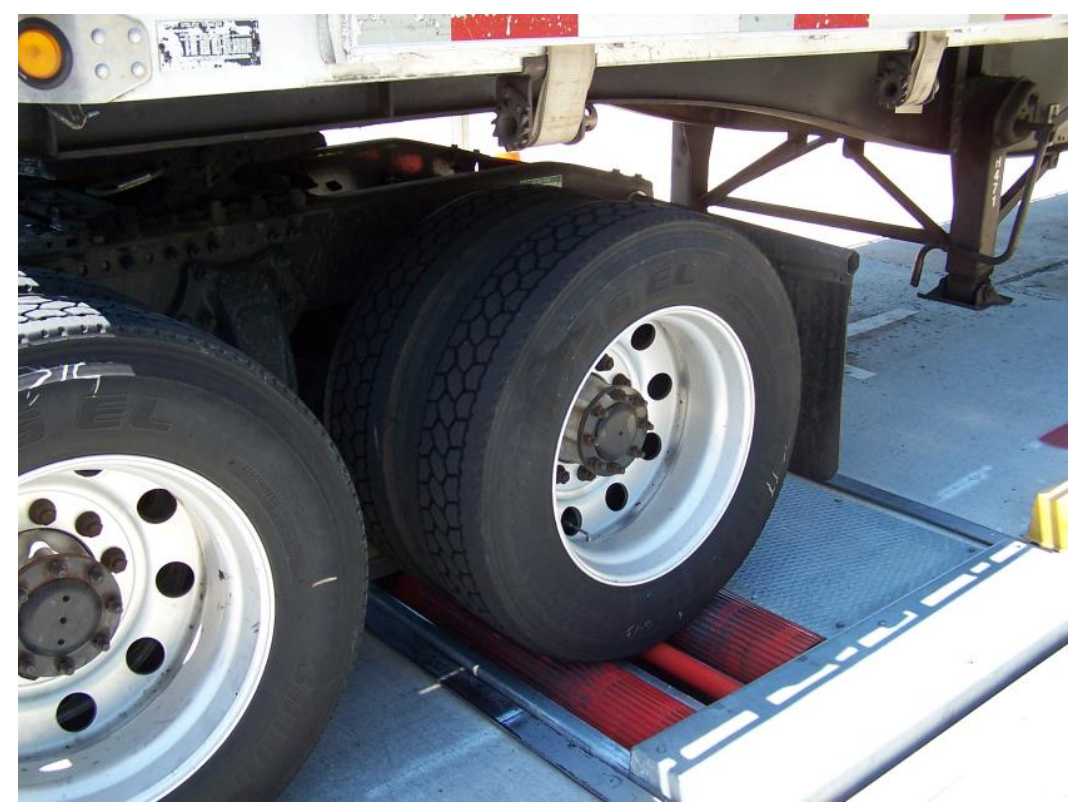

Figure 9. Performance-based brake tester located at the Greene County Inspection Station (rollerdynamometer type).

The PBBT also has the capability to record brake application pressure during the test. Data files are generated for each test. These files include the streaming pressure, BF, and weight data, as well as other header and label information.

\subsection{TESTS PERFORMED}

Several different types of data were collected during the testing task of this project. Constant-pressure stopping tests were conducted at several different combinations of brake application pressure $(15,20,25$, and $30 \mathrm{psi}$ ), load conditions (moderately and fully laden), and speeds (20 and $30 \mathrm{mph}$ ). Data was collected at $10 \mathrm{~Hz}$. Both standard and stepped-pressure PBBT tests with brake pressure transducers were performed for each loading condition. The stepped-pressure test included the constant-pressure intervals of brake application at 15, 20, 25, and 30 psi. The PBBT data files included $10 \mathrm{~Hz}$ streaming data collected during the testing of each axle. Two weeks of real-world duty cycle (driving and braking) data were also collected at $10 \mathrm{~Hz}$.

\subsubsection{Stopping Tests}

One type of testing performed as part of this research project included constant pressure stopping tests to provide "ideal" data to determine the pressure-BF relationship. For these tests, the loaded vehicle was brought up to a pre-determined speed, and then a constant brake application pressure was applied until the vehicle came to a complete stop. These tests were repeated for two different loading conditions [moderately loaded (approximately 50\% load capacity) and heavily loaded (about $90 \%$ load capacity)], and from two different speeds ( 20 and $30 \mathrm{mph}$ ). Pressures tested were 15 psi to $30 \mathrm{psi}$, in 5-psi increments. The gross vehicle and axle weights in pounds for the stopping tests are shown below in Table 1. 
Table 1. Vehicle Weights for Stopping Tests

\begin{tabular}{lcc}
\hline & $\begin{array}{c}\text { Medium Load } \\
(\mathbf{l b s})\end{array}$ & $\begin{array}{c}\text { Heavy Load } \\
(\mathbf{l b s})\end{array}$ \\
\hline Steer Axle & 9,180 & 9,390 \\
Drive Axle & 14,560 & 20,480 \\
Trailer Axle & 9,150 & 14,150 \\
\hline Total $(\mathbf{G V W})$ & $\mathbf{3 2 , 8 9 0}$ & $\mathbf{4 4 , 0 2 0}$ \\
\hline
\end{tabular}

Each test scenario (given combination of starting speed, brake application pressure, and loading condition) was performed two to four times. These tests were performed on two separate days, one with a heavy load, the other with a medium load. The stopping tests were performed at Greene County Inspection Station's eight-acre parking lot under supervision of the Tennessee Highway Patrol. Vehicle weight tickets were obtained at the beginning and ending of each day of testing as well to confirm the Air-Weigh and PBBT weight readings.

\subsubsection{Standard and Modified PBBT Tests}

PBBT tests were also part of the data collection. At the beginning and ending of each day of testing, a regular PBBT test with pressure transducers was conducted. In these tests, the driver slowly, consistently increased the brake application pressure until wheel lock occurred or a certain value of BF was reached. The PBBT score is the maximum BF experienced in the test (sum of all wheel ends) divided by the gross vehicle weight $(\mathrm{GVW})$. The pressure/time trace looks similar to that shown in Figure 10.

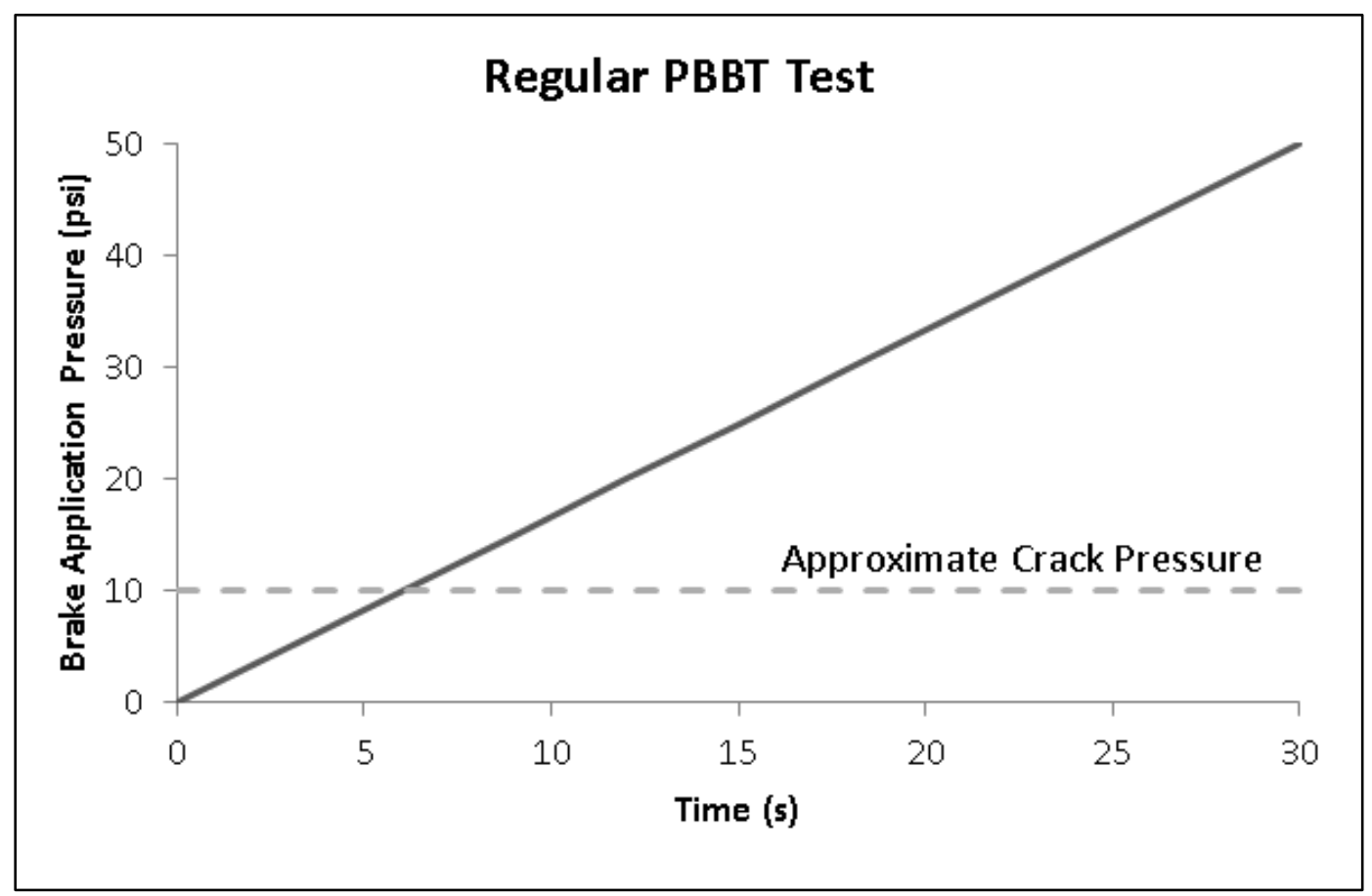

Figure 10. Conceptual trace of brake application pressure for regular PBBT test.

In addition, modified "stepped-pressure" PBBT tests were also conducted. These tests were set up as regular PBBT tests with pressure transducers; however, instead of slowly increasing the brake application to maximum, the brakes were held at certain brake application pressures for several seconds using the in- 
cab display to provide feedback to the driver. This provided a more accurate reading of corresponding BF from the PBBT for these discrete pressures $(15,20,25$, and $30 \mathrm{psi}$ as for the stopping tests). The pressure-time trace for such a test looked similar to that shown in Figure 11.

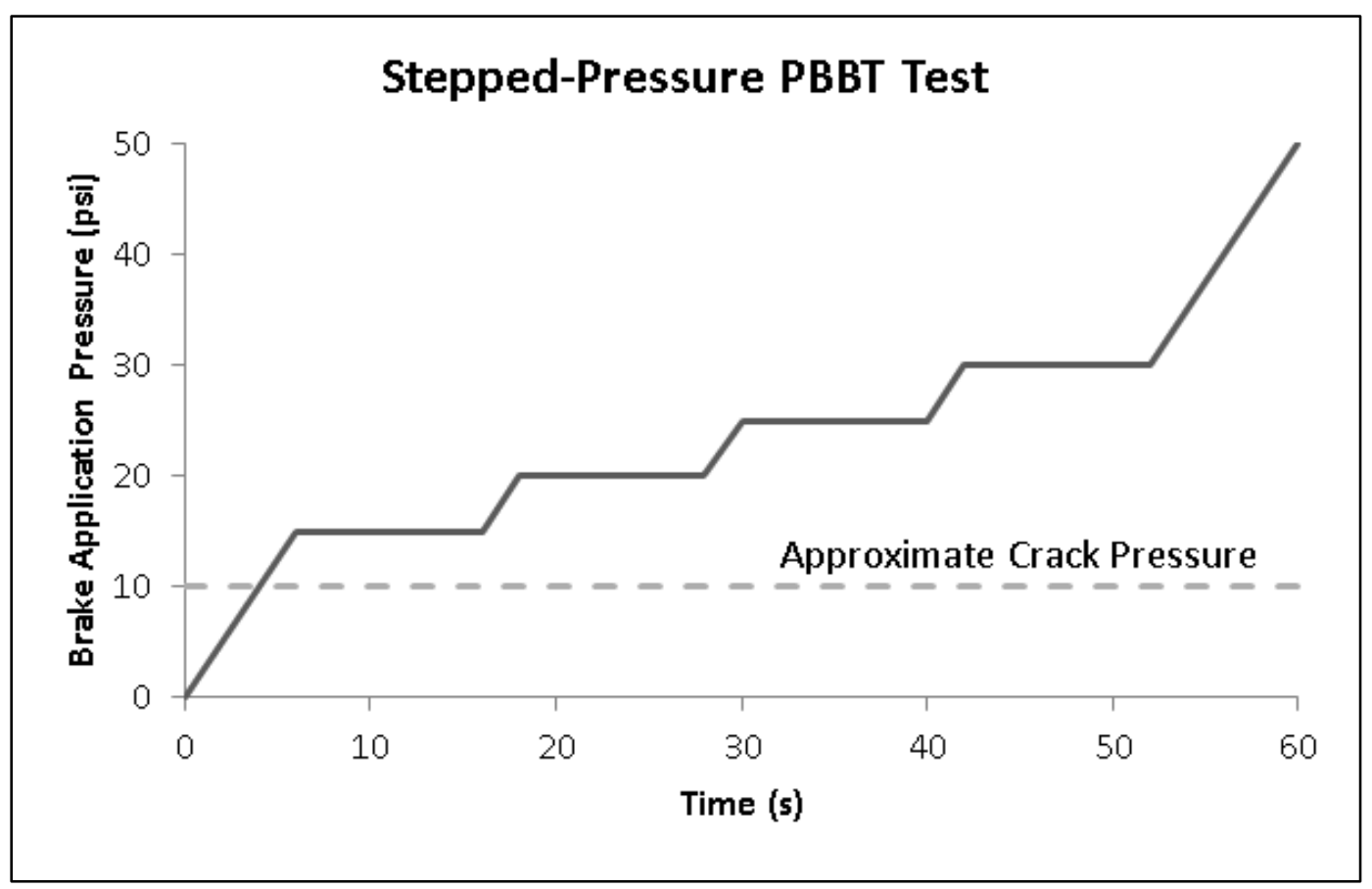

Figure 11. Conceptual trace of brake application pressure for stepped-pressure PBBT test.

Due to the data file limitations of the PBBT machine, each modified PBBT test had two test components: the first test involved dwells at 15 and 20 psi, the second at 25 and 30 psi. Both types of PBBT tests performed at the beginning and ending of each test day. Thus, two of each type of PBBT test were performed for each loading condition.

The pressure information from the PBBT test is slightly different from the pressure information recorded by the DAS (and shown on the in-cab display). The MGM brake application pressure sensor is located at the foot/treadle valve, and the PBBT pressure sensor is located at glad-hand connector between the tractor and trailer. In addition, no effort was made to calibrate or determine the error offset of either system. By collecting this data with the PBBT machine as the driver used the in-cab display to regulate air pressure, the relationship between the pressures at these two locations could be examined.

\subsubsection{Normal Vocational Operations Data}

The same 10-Hz data collected during the stopping tests was collected during the test vehicle's regular operations over a two-week period. This "real-time" data is the type of data which would ultimately be used by a fully-developed on-board system. 


\section{ANALYSIS AND RESULTS}

\subsection{DATA FROM PRESCRIBED TESTING}

The purpose of the scripted testing (stopping test data and PBBTs) was to provide data to understand trends and relationships from "clean" data with limited variables. The trends apparent from this data provide insight into the processing and interpretation of the real-world duty cycle data.

\subsubsection{PBBT Test}

Force-pressure data was extracted from the stepped-pressure PBBT test by detecting intervals where change in brake application pressure was very small. These intervals are identified in the streaming data by the dotted lines in Figure 12.

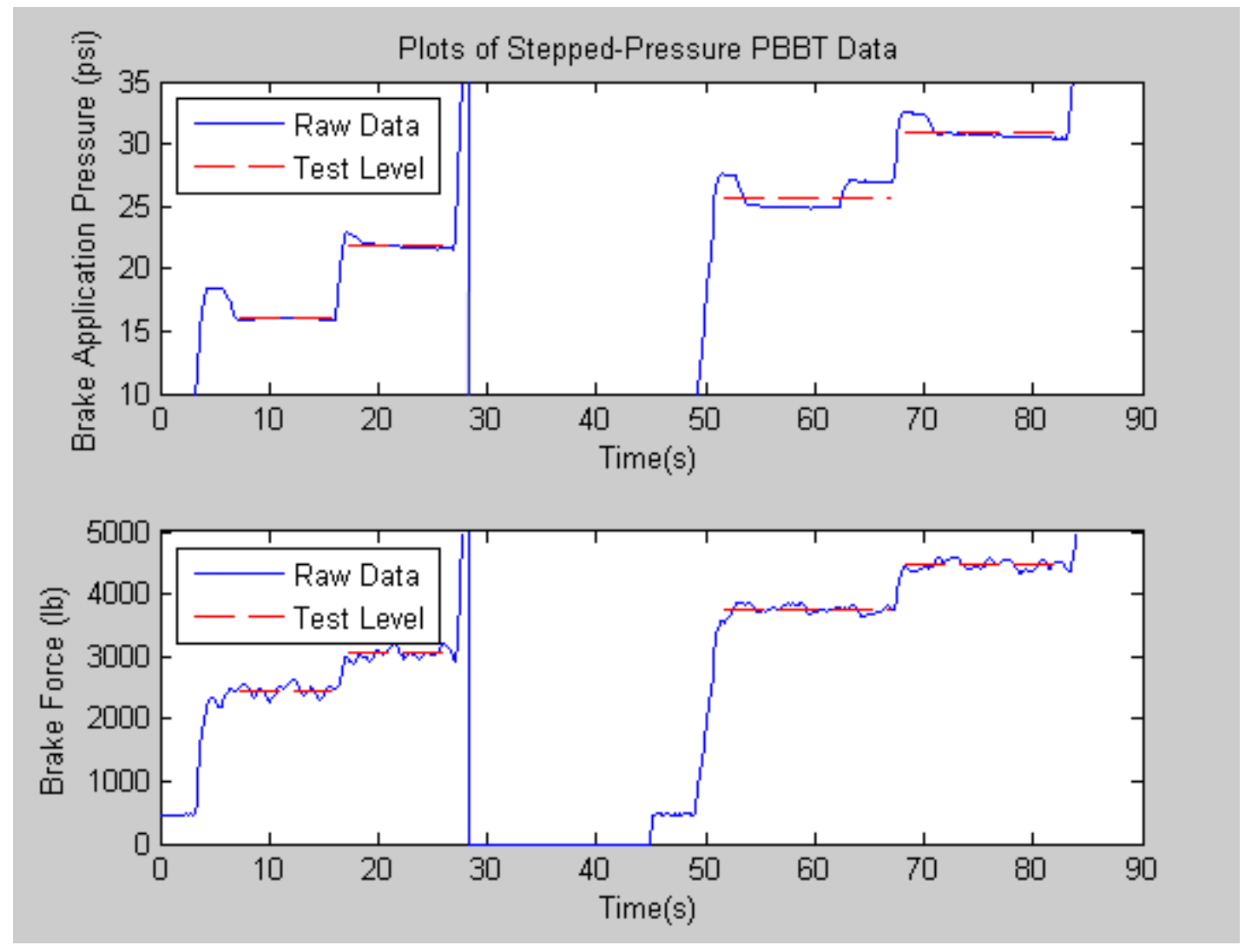

Figure 12. Data from stepped-pressure PBBT test.

Each constant-pressure interval provided a point on the P-BF curve by averaging both the brake application pressure and BF over these intervals. The information extracted from the PBBT data shown in Figure 12 is plotted in Figure 13. 


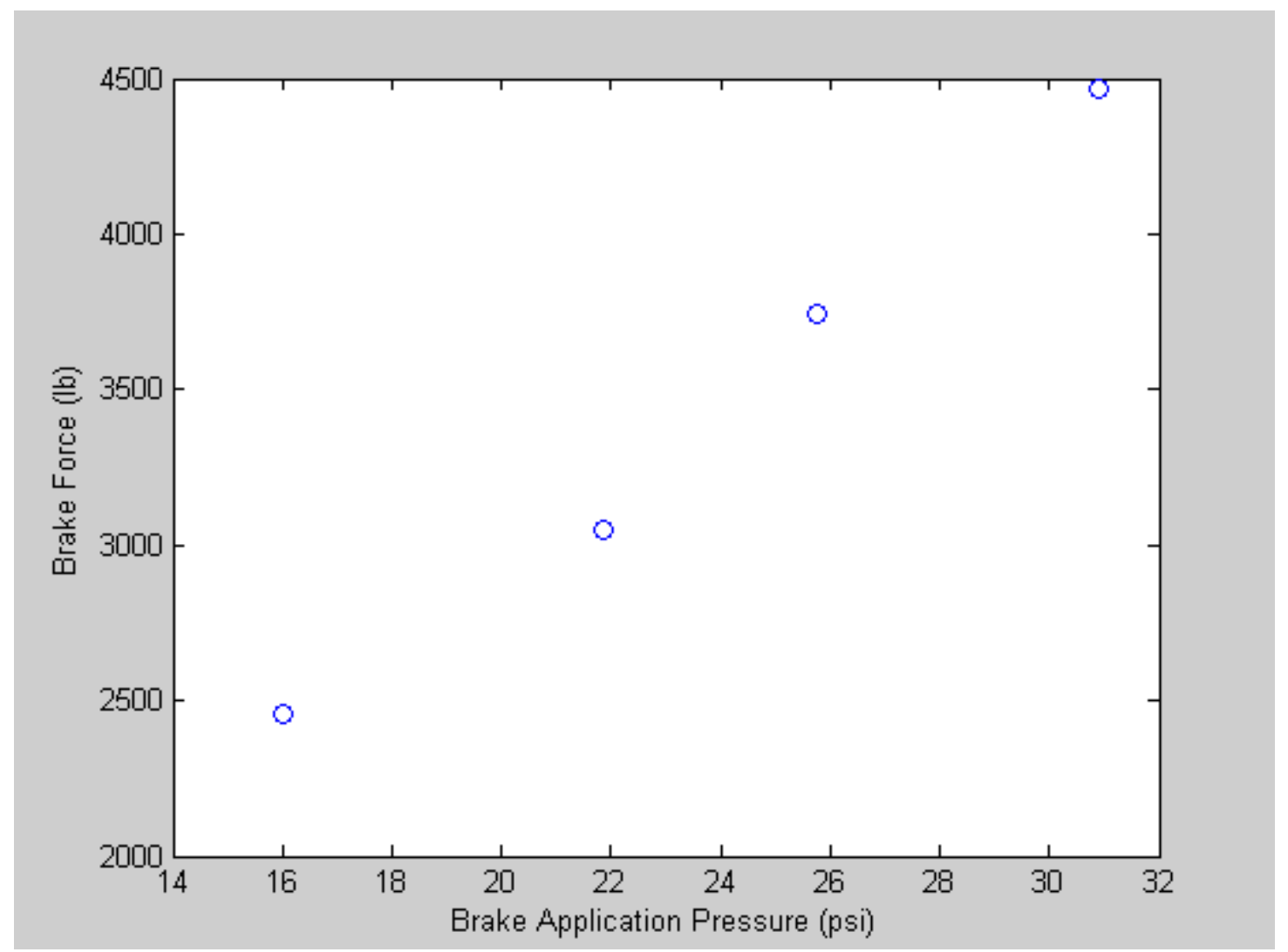

Figure 13. Brake application pressure and brake force extracted from stepped-pressure PBBT test.

As apparent from this graph, there is a strong linear relationship between the brake application pressure and the BF in the 15-30 psi region in which the modified PBBT tests were conducted.

As mentioned previously, the pressures recorded by the PBBT's pressure transducer at the glad-hand connector were slightly different from those recorded by the DAS from the pressure transducer at the foot valve and shown in the in-cab display used during testing. The data from each of these sources is plotted in Figure 14; note that the in-cab display showed pressures rounded to the nearest whole psi, while a finer pressure resolution was available from the PBBT data file. 


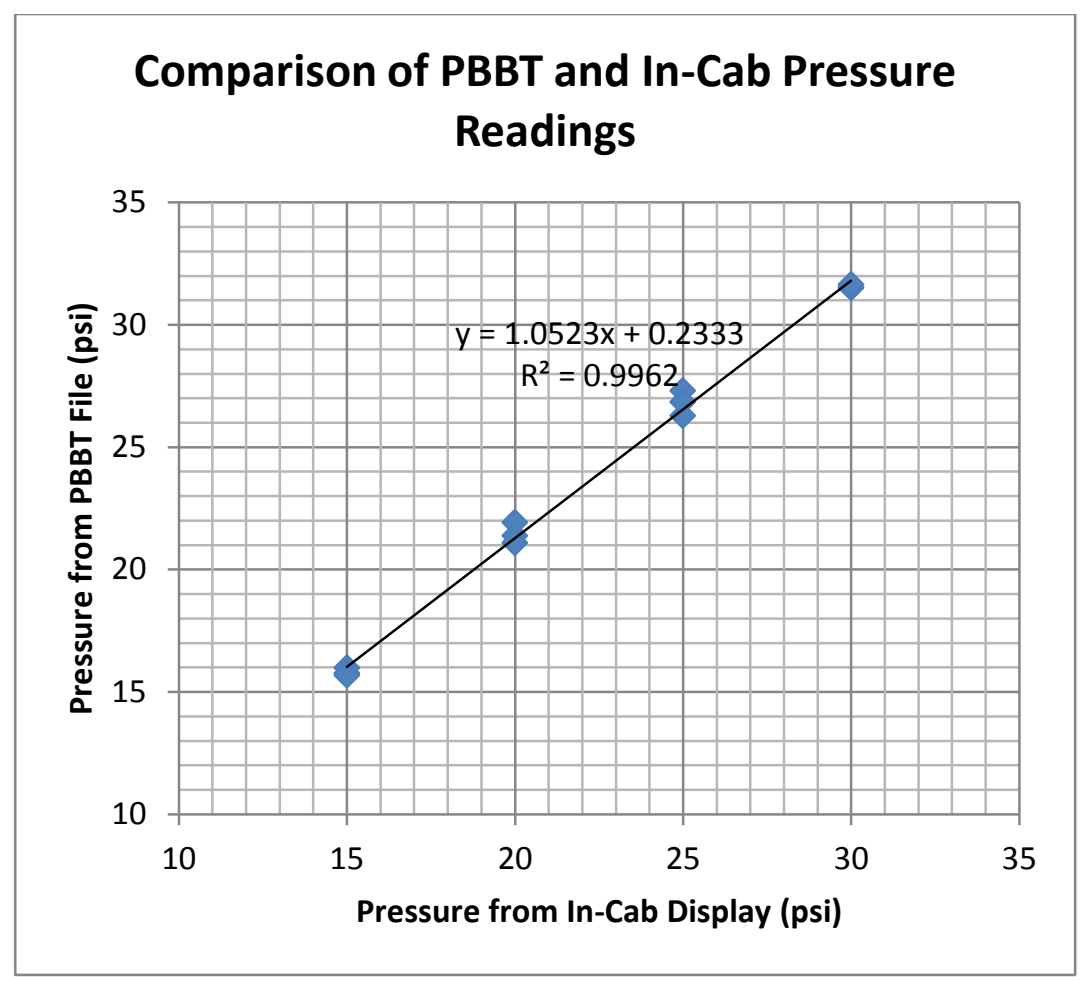

Figure 14. Comparison of pressure readings.

This plot reveals that although these two sources of brake application pressure are not identical, the differences between the two are negligible for the purposes of this study. Figure 15 compares the P-BF relationship from the stepped-pressure PBBT test to that of the regular PBBT test for the two different loading conditions. 


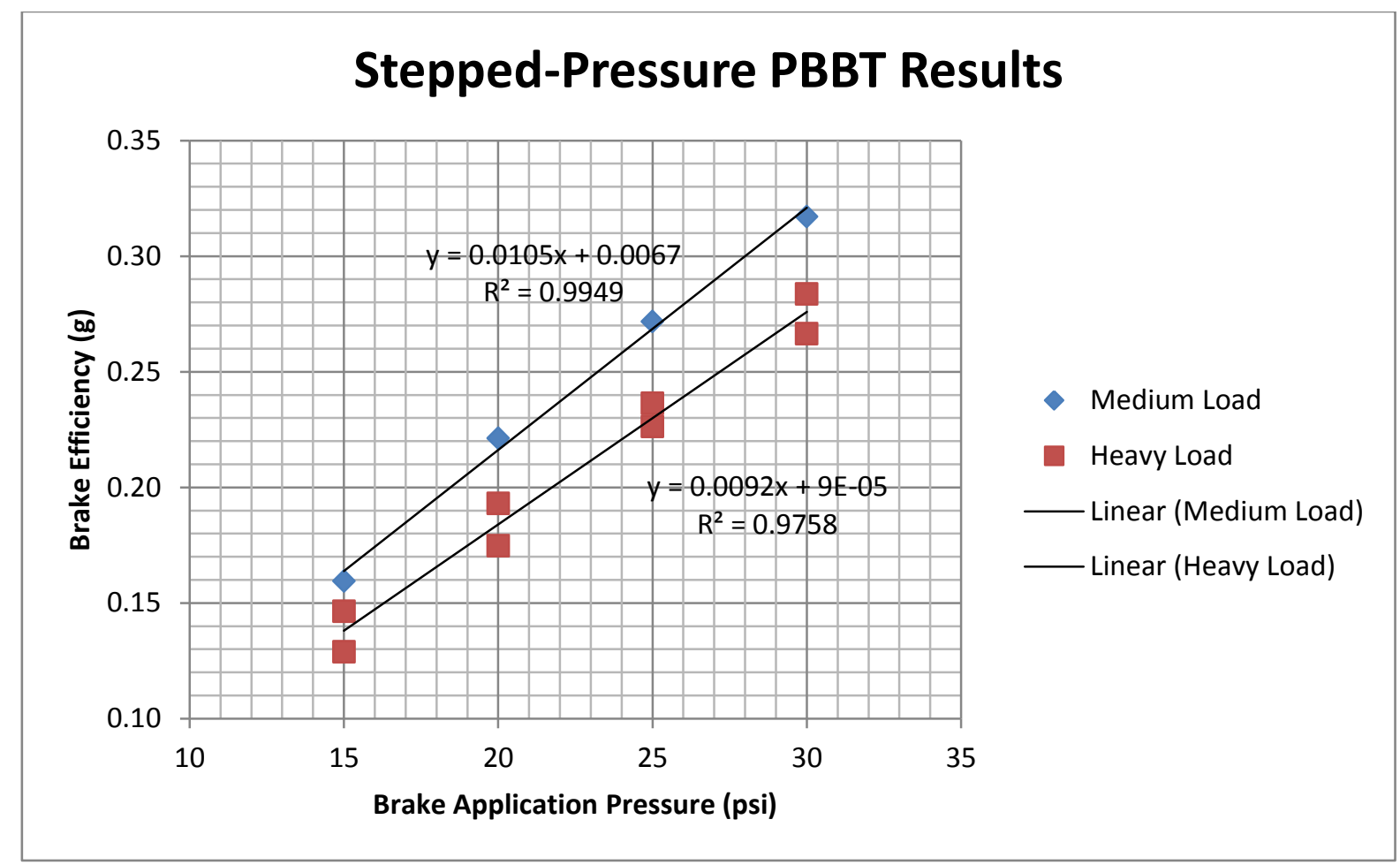

Figure 15. Comparison of stepped-pressure PBBT results for two loading conditions.

An important conclusion that can be drawn from this plot is the effect of weight on the P-BE relationship, particularly in the 15-30-psi region where this research was focused. Compared to the heavy load situation, this line is higher on the plot for the medium load condition. This is to be expected because BE is the total braking force) divided by the GVW; the $\mathrm{BF}$ is not expected to change significantly from one load to another.

\subsubsection{Stopping Tests}

The stopping tests performed at Greene County were designed to allow a data extraction algorithm to be developed to construct the BE-pressure profile from the real-world data. Because the stopping tests performed at Greene County were carefully scripted, the pressure and deceleration traces are smooth, as shown in Figure 16. 


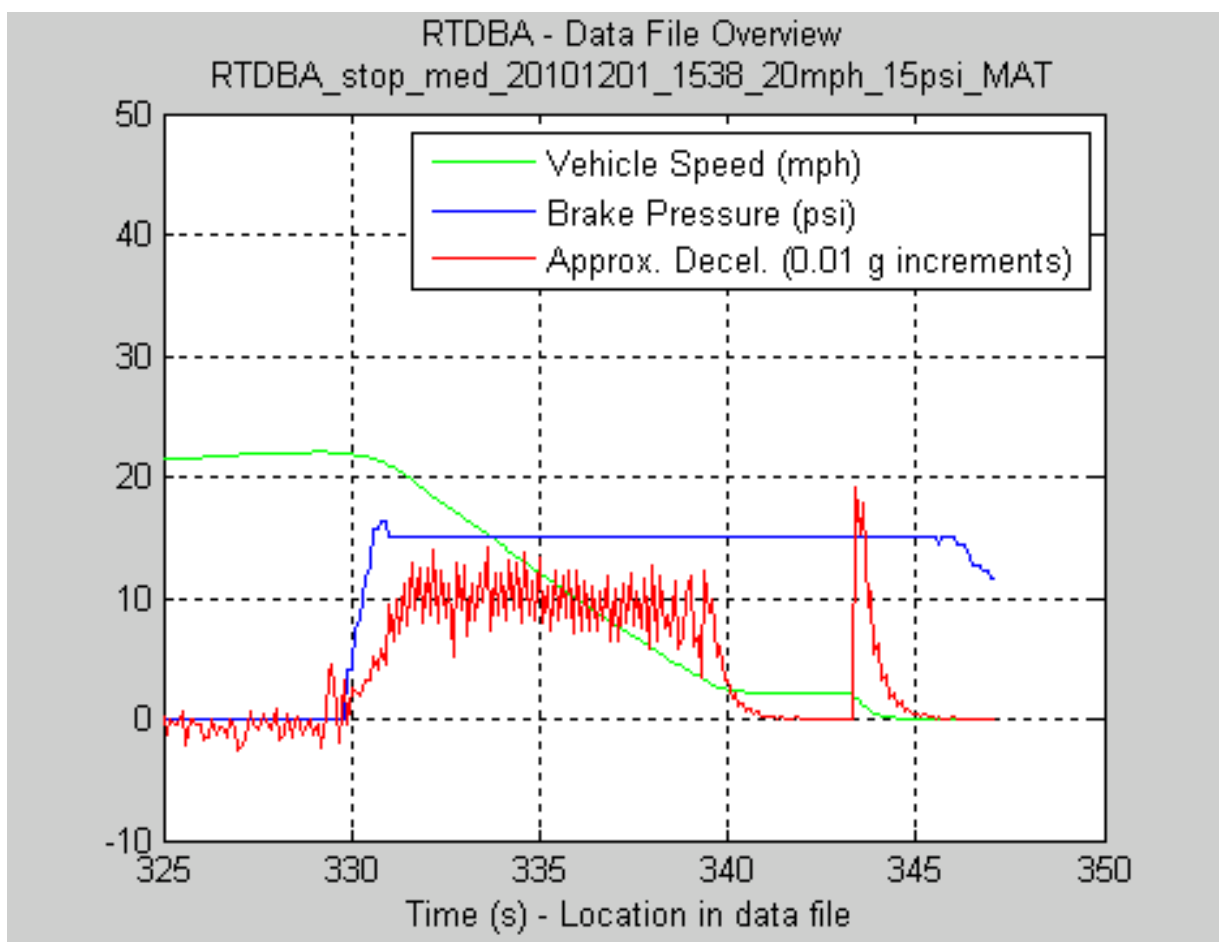

Figure 16. Sample data from stopping test.

Figure 17 compares the stopping test results for stops performed from the two different test speeds, 20 and $30 \mathrm{mph}$. 


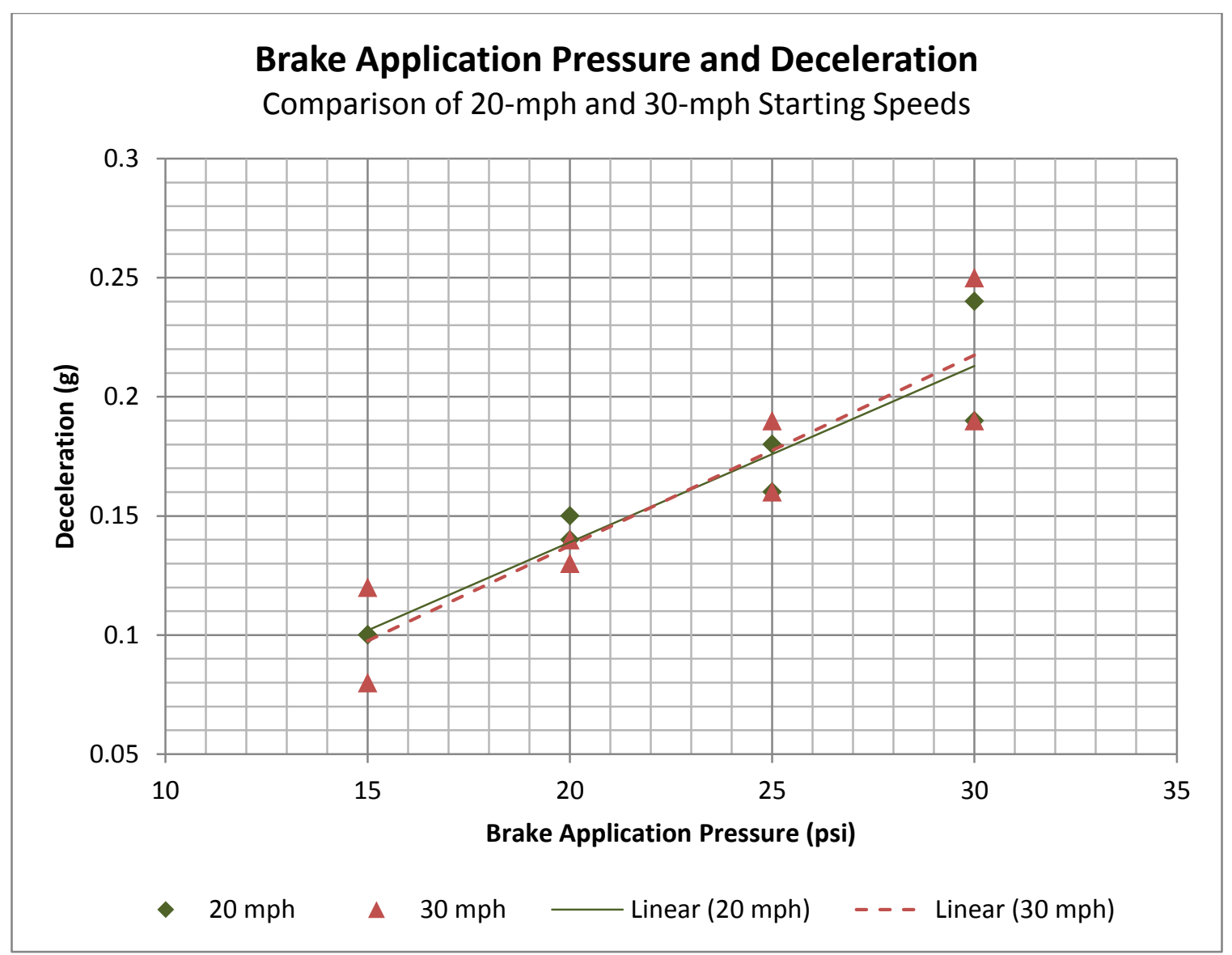

Figure 17. Effect of starting speed on deceleration-pressure profile.

From these graphs it is apparent that starting speed has no appreciable effect on deceleration (provided the brake application pressures are consistent. This is consistent with the theory discussed previously, where deceleration was assumed to be a function of weight and brake application pressure only, in the absence of an appreciable road grade.

Initially, plots of the stopping tests were generated in order to visually estimate the BE (deceleration) for each brake application pressure and load condition. This data is shown along with the other test data from the PBBT tests in Figure 18 and Figure 19. 


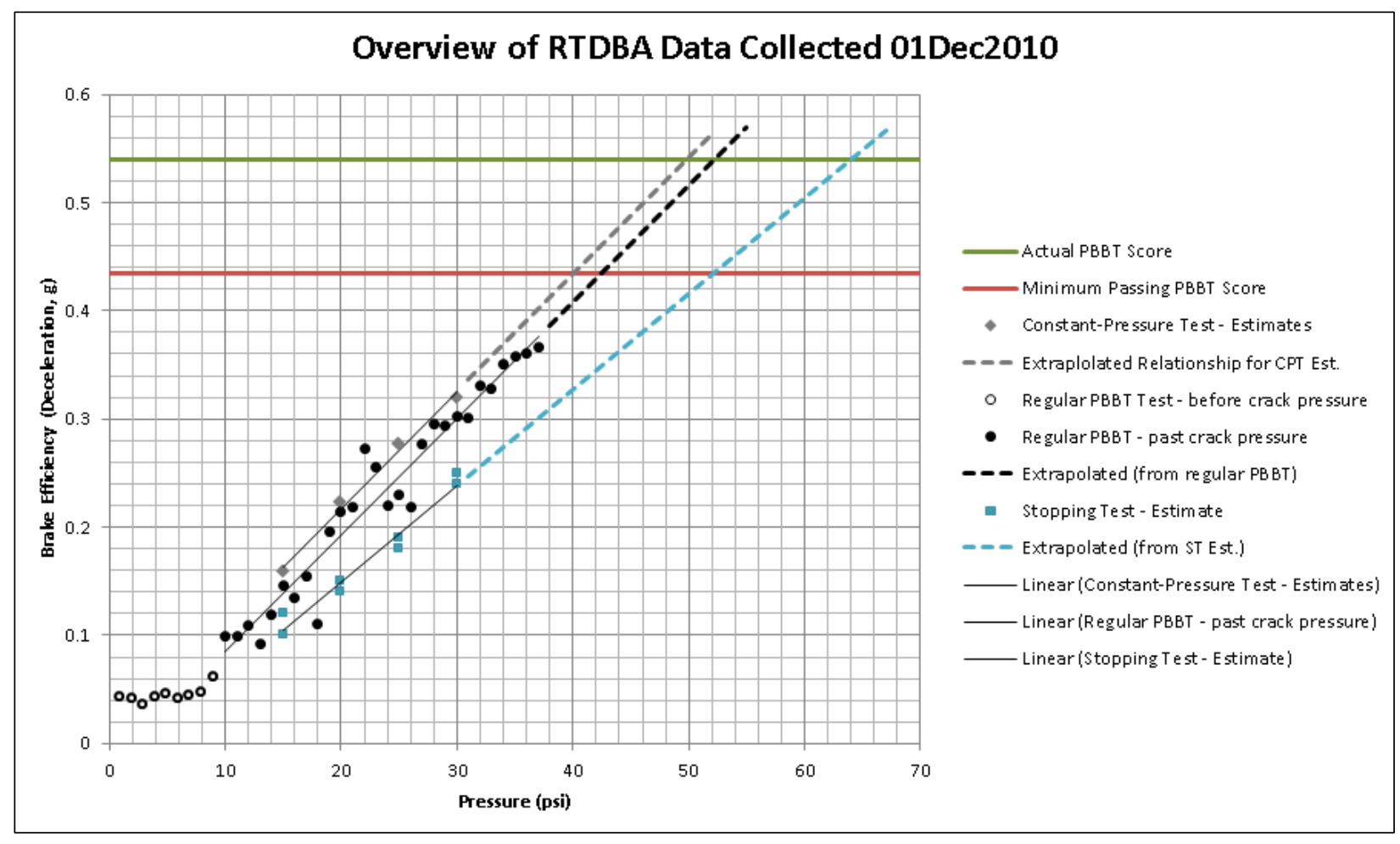

Figure 18. Summary plot of medium-load test data. 


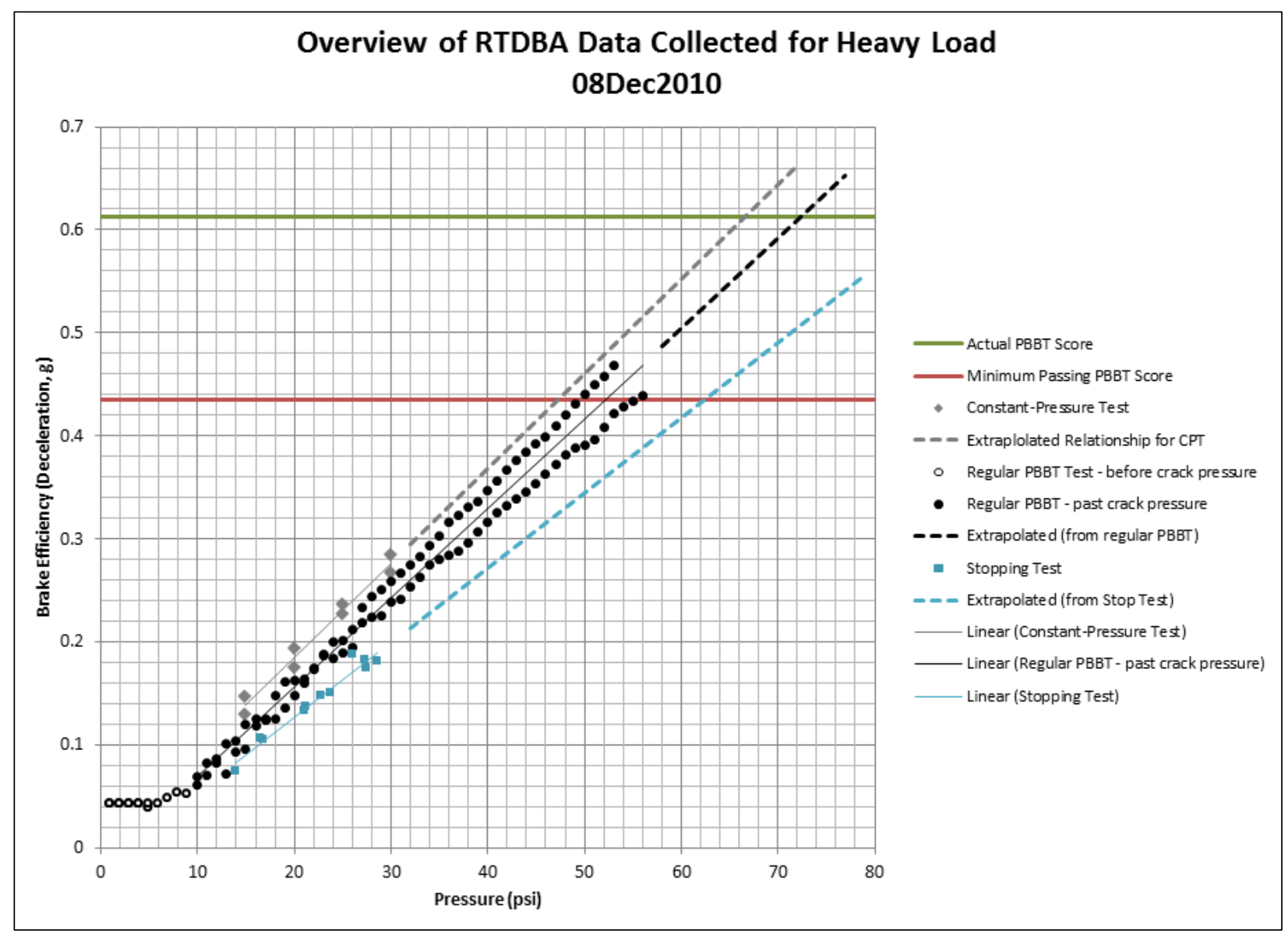

Figure 19. Summary plot of heavy-load test data.

In both loading cases, the stopping test data trend line is below the PBBT data, but there remains a strong linear correlation between the BE and the pressure. The stopping tests have a lower deceleration than PBBT-measured BE for a given pressure; this is consistent for heavy or medium loads, which showed the same relationship as seen in the PBBT results. These plots also show the actual PBBT score for each of these tests. Note that the plotted points from the PBBT test do not reach the actual PBBT score, indicated by the solid green line at the top of the graph. This is because PBBT tests are performed one axle at a time. The pressures shown were reached by all brake positions tested. Thus, the maximum pressure plotted for PBBT test data corresponds to the maximum pressure experienced by the wheel-end which locked up first (at the lowest pressure compared to the other wheel-ends). 


\subsubsection{Implications for Real-World Data}

Analysis of the test data collected led to a number of key findings listed below.

- The difference between the pressure at the foot valve and the pressure at the PBBT transducer was found to be minimal.

- There was found to be no noticeable difference in the P-BF relationship as a result of different starting speeds for a given loading condition; this supports the theory behind the development of an on-board system.

- A strong linear relationship was found between the brake application pressure and deceleration in the tested region for all types of tests (regular PBBT, stepped-pressure PBBT, and stopping test).

- A difference was identified between the stopping test results and the PBBT results. Decelerations from the stopping test were consistently lower than the equivalent PBBT measurements for a given pressure. While this may point to additional deceleration forces present in real stopping situations, such additional forces are clearly consistent and do not preclude the identification of trends in the actual stopping data.

- Also in agreement with the background theory, loading was found to be a significant factor in stopping performance. This was expected because the applied braking force for a given pressure is relatively constant for the vehicle's braking system (with the exception of wear or other problems introduced). Deceleration is proportional to mass; therefore, increased weight causes a proportional decrease in corresponding deceleration for the same braking force.

The preceding graphs have shown the relationship between brake application pressure and BF to be approximately linear in the region of routine brake applications (approx. 15-30 psi). From Newton's second law $(\mathrm{F}=\mathrm{ma})$, the deceleration for a given brake application pressure (corresponding to a given brake force) is proportional to the GVW. Therefore, the relationship between brake application pressure and deceleration for normal, properly-functioning brakes can be represented by the family of curves shown in Figure 20. In this figure, each trace represents a different loading condition. 


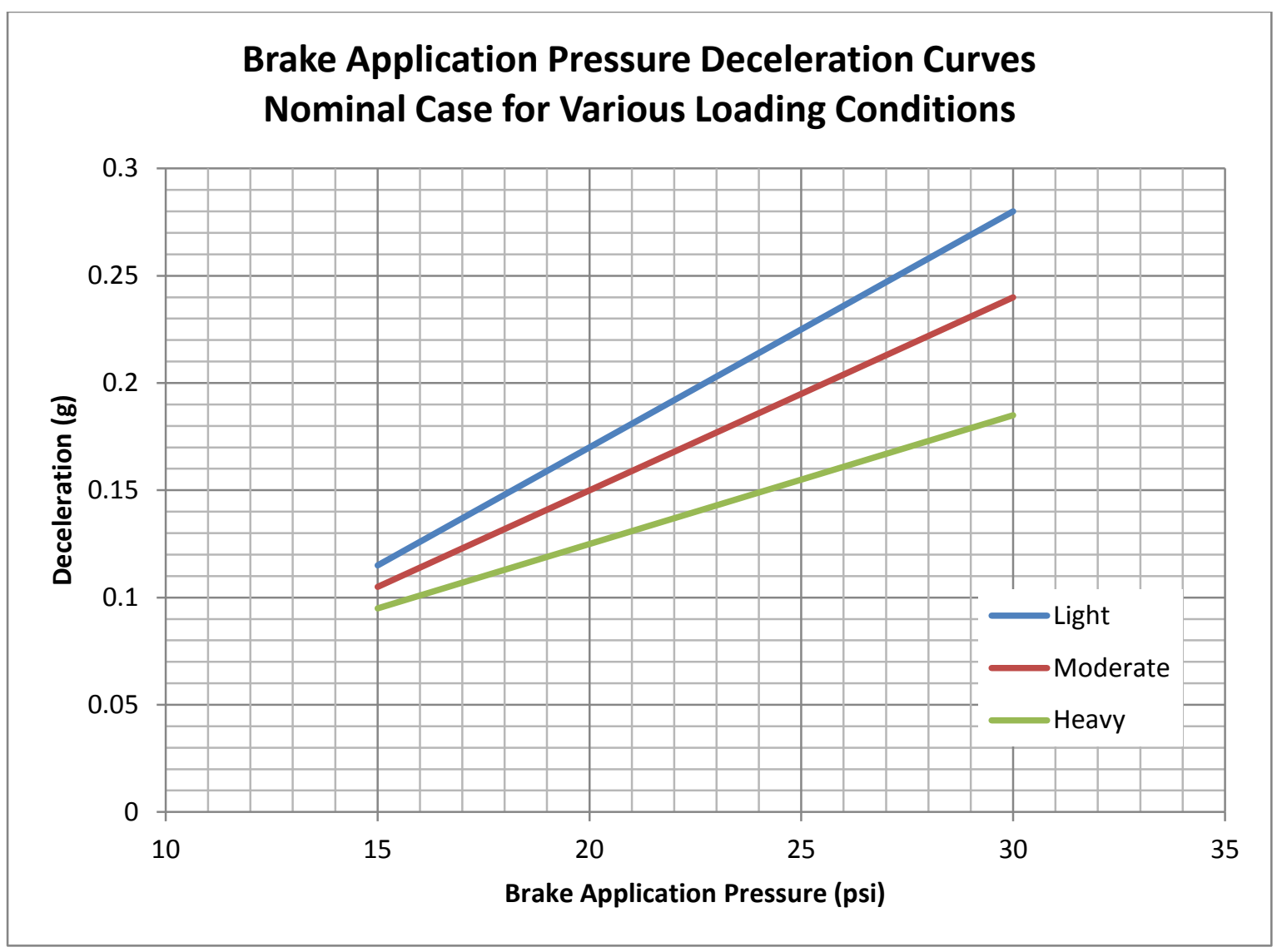

Figure 20. Conceptual plot of effect of loading conditions on pressure-deceleration relationship.

It is anticipated that significant changes in characteristics of the above family of curves would be indicative of major problems with the braking system. For example, if a truck's brakes experience significant degradation due to wear or are inoperative, it is expected that the braking system would not be able to achieve the same level of deceleration for a similar loading condition; this would be indicated by a reduction in the slopes of the lines shown. Similarly, an air distribution problem may mean that additional pressure at the foot valve which activates the brakes; this might be apparent from a shift of the curves shown above toward higher pressure. Two sample possible scenarios are shown in Figure 21. To avoid confusion, only a single loading condition is shown; it is expected that the traces for the other loading conditions would be similarly affected. 


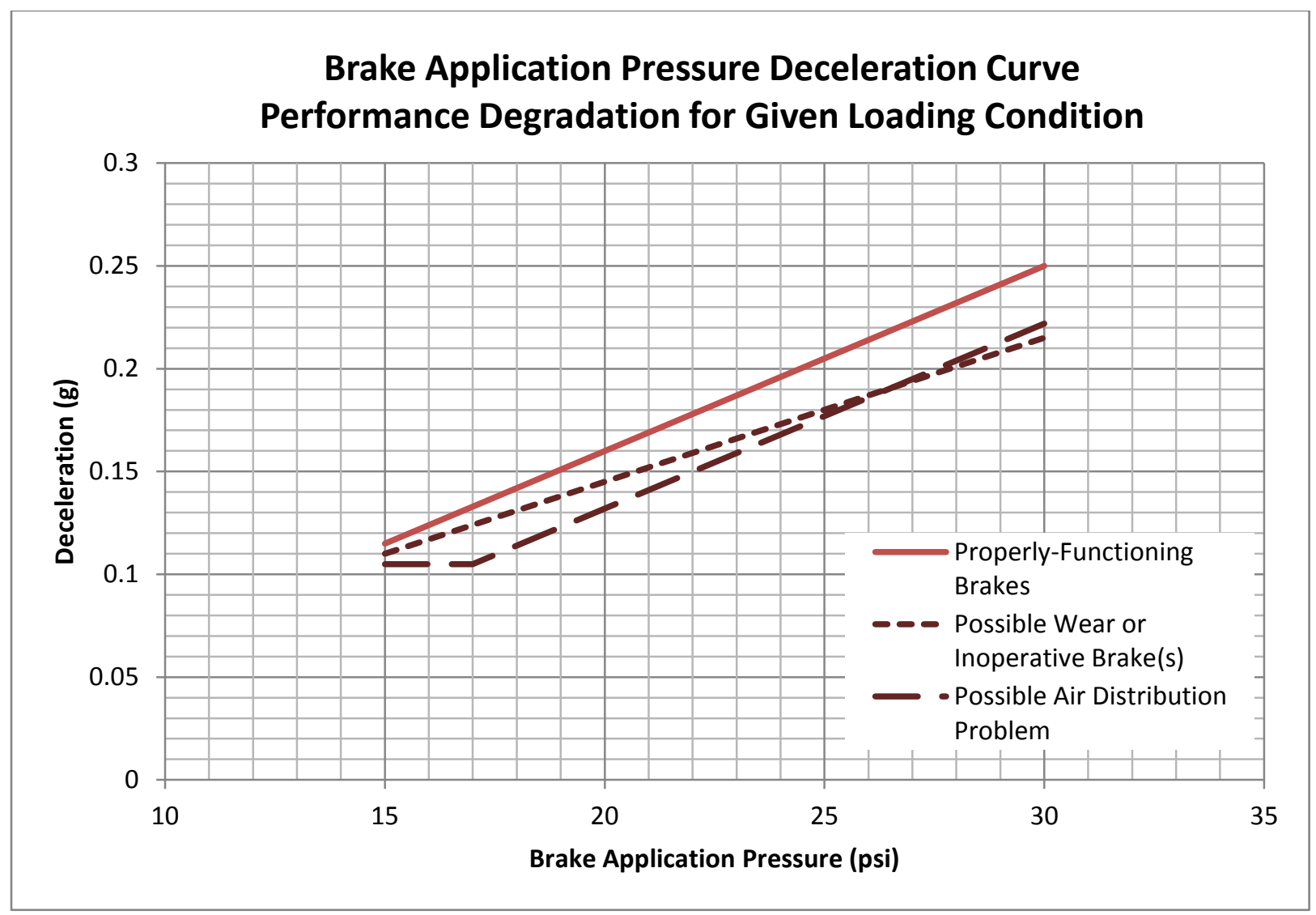

Figure 21. Conceptual plot of evidence of braking system problems on pressure-deceleration plot.

Thus, degradation might be apparent from a variety of changes in the family of curves. The curve for a given loading condition may be no longer linear. Alternatively, the slope of the curve for a given loading condition may decrease. Another possibility is that that the curve(s) indicate that the braking system cannot achieve a minimum deceleration such as might be required to pass a PBBT test.

\subsection{DUTY CYCLE DATA}

The real-world duty cycle data was examined to confirm the two weeks of collected data contained sufficient information of the type needed to support the analysis outlined previously. Then, an algorithm was developed to extract stopping test data from duty cycle data to determine the relationship between pressure and deceleration based on real-world data.

\subsubsection{General Characteristics}

Approximately two weeks of regular duty cycle data was collected at the increased rate of $10 \mathrm{~Hz}$. The speed distribution for this data is shown in Figure 22. 


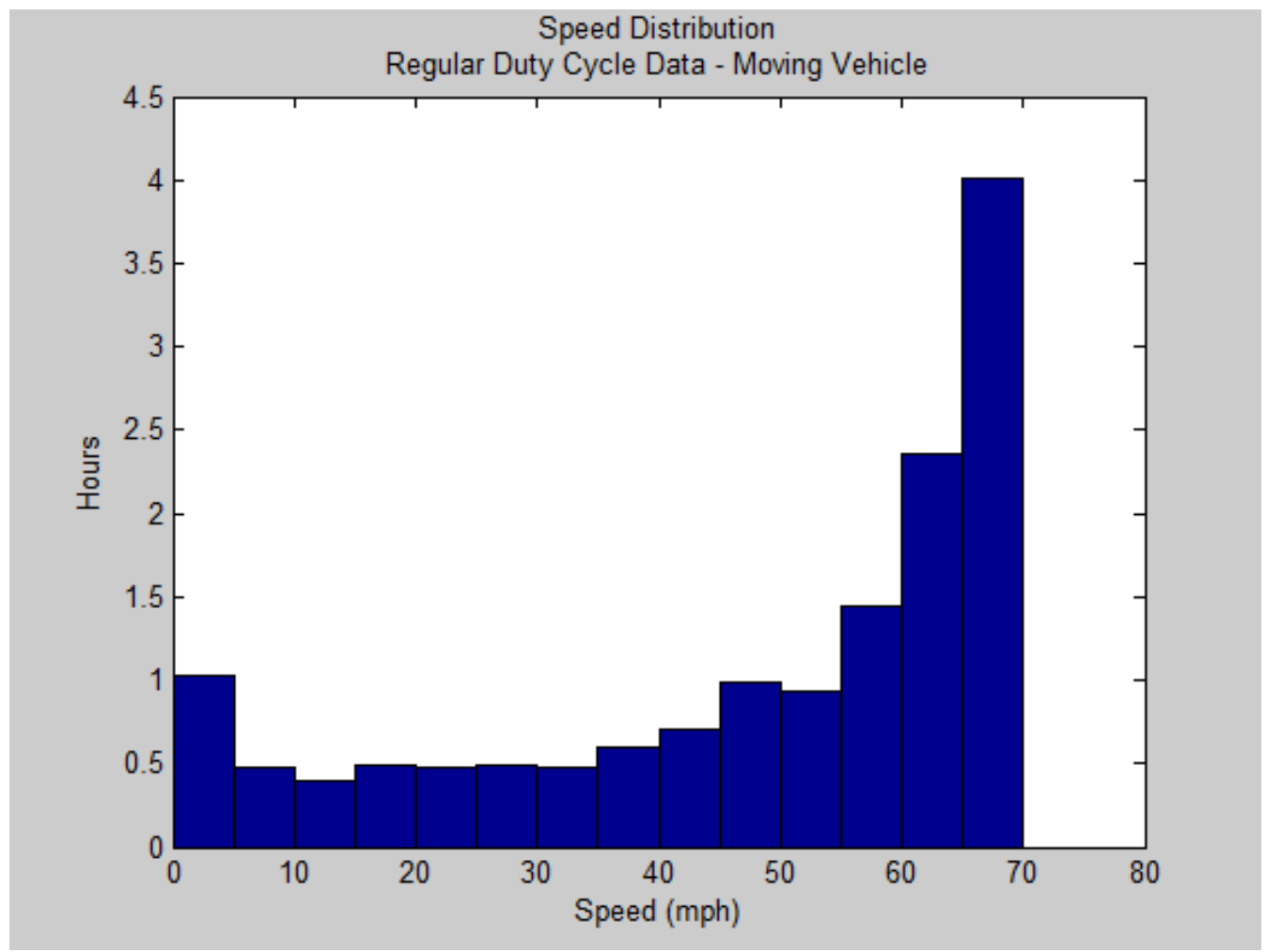

Figure 22. Speed distribution of regular duty cycle data.

As expected, the pressure distribution of this data showed the vast majority of braking events occurring below 30 psi (Figure 23). This is consistent with the larger set of data collected at the lower 1-Hz update rate (Figure 5). 


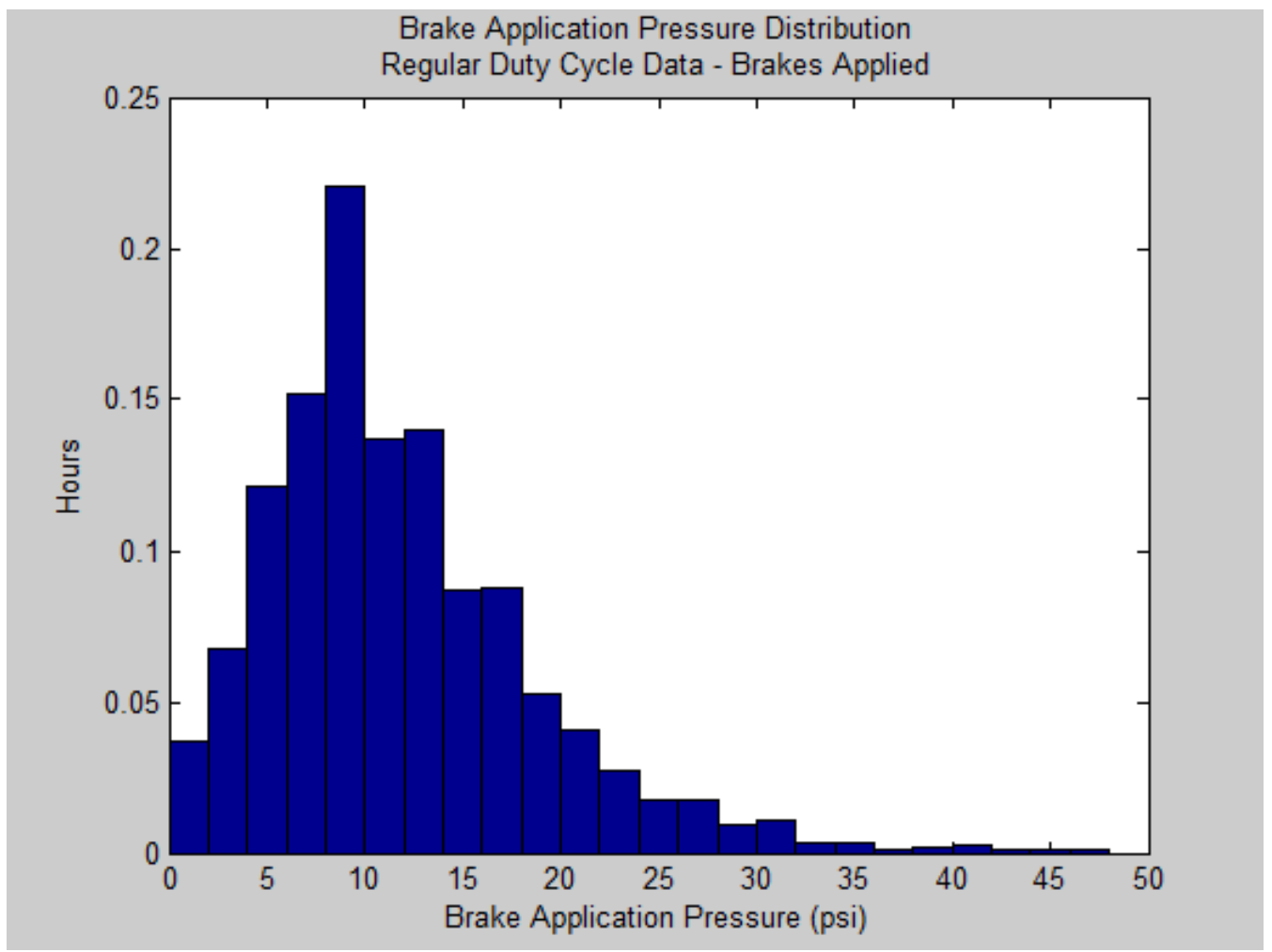

Figure 23. Distribution of brake application pressures (greater than zero) in duty cycle data.

As shown in Figure 24, the weight supported by the steer axle remained virtually unchanged throughout the course of the data collection period. Therefore, it is unlikely that the weight on this axle would need to be incorporated into a brake performance algorithm as it contributes very little to the wide variation in GVW. The load on the drive axle, however, did vary considerably under various loading conditions, indicating both low and moderate loads (Figure 25). 
Regular Duty Cycle Data - Valid Data Readings

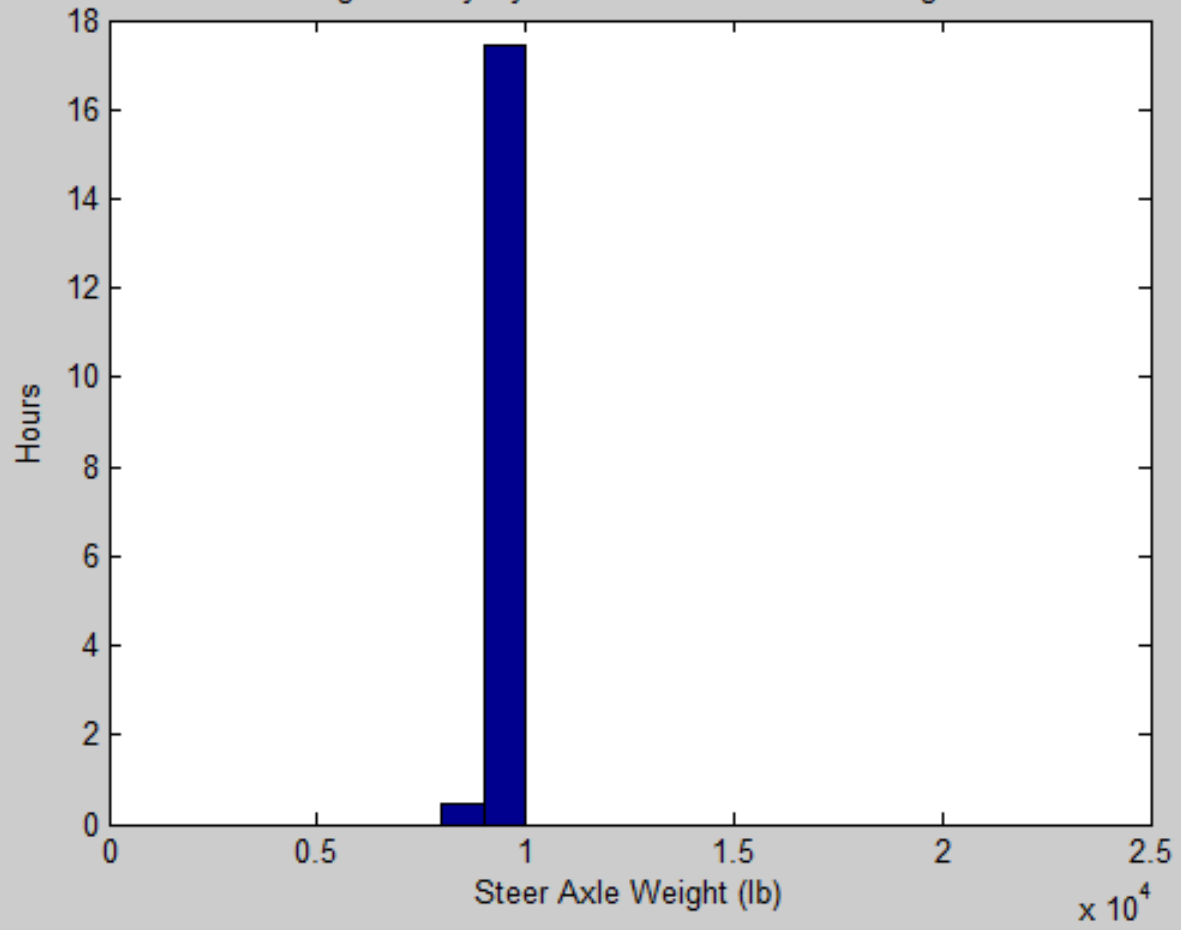

Figure 24. Distribution of steer axle weight in duty cycle data.

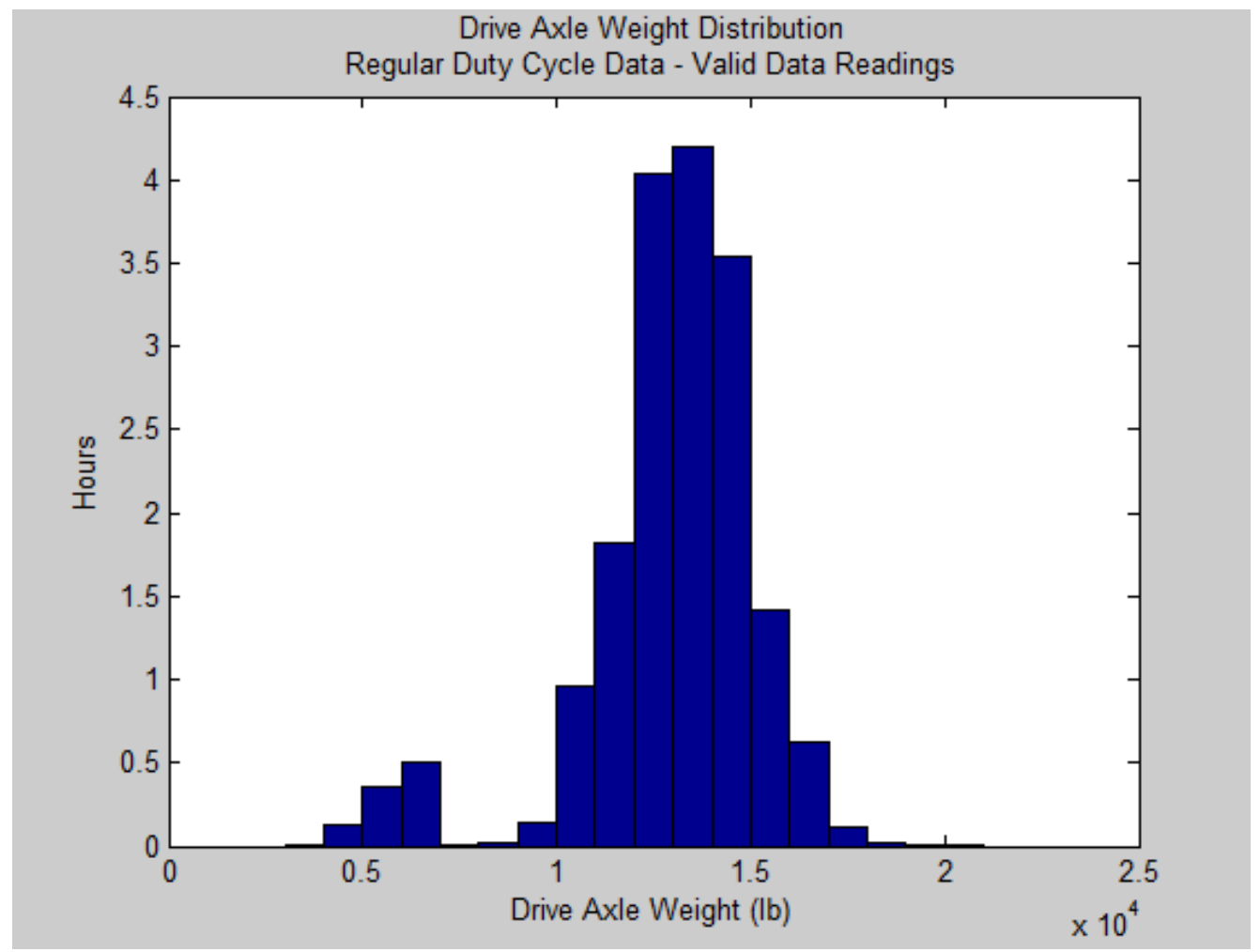

Figure 25. Distribution of drive axle weight in regular duty cycle data. 
A two-dimensional histogram was generated to aid in the development an algorithm for brake performance monitoring that incorporates several representative speeds and decelerations (Figure 26).

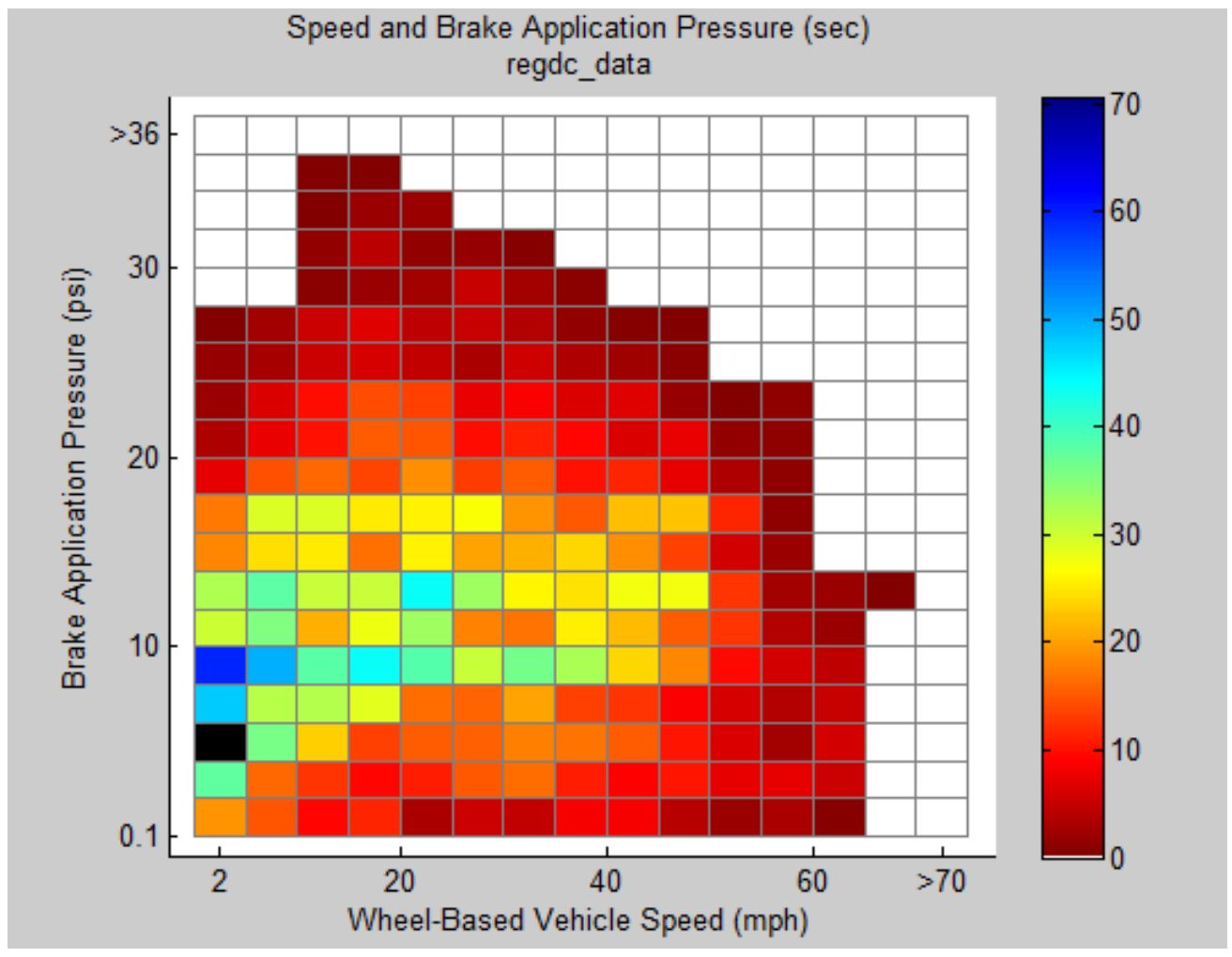

Figure 26. Bivariate speed and pressure distribution in regular duty cycle data.

In this figure, the color of the square indicates the proportion of duty cycle time spent at that given speed and pressure. From this graph, we can see that braking events occur at speeds across the full speed range (up to almost $70 \mathrm{mph}$ ), and most braking events occur below approximately 25 psi. This indicates that stopping data will be available in the data stream for a variety of speeds across the low pressure ranges studied in this effort (15-30 psi).

\subsubsection{Extraction of Braking Data}

Unlike the scripted constant-pressure stopping tests, real-world stops were not characterized by a nearconstant brake application pressure. Thus, an algorithm needed to be developed to extract braking events from this real-world data. The information collected during the tests conducted at the Greene County, Tennessee Inspection Station was screened to extract only the most relevant and valid data as described by the basic theory presented previously. The data collected contained many "braking events" that qualified as valid data (i.e., events in which the average brake application pressure, $\boldsymbol{B A P r}$, was greater than $10 \mathrm{psi}$ and the vehicle speed at the beginning of the brake application was greater than $5 \mathrm{mph}$ ).

Figure 27 shows one such braking event that was part of the medium-load tests. The figure presents the brake application pressure profile and the vehicle speed profile. The average $\boldsymbol{B A P r}$ for this event, which lasted about 9.5 seconds, was 19.71 psi. The driver maintained a $\boldsymbol{B A P r}$ of roughly 23.5 psi for about 5.2 seconds (5,376.4 to 5,381.6 seconds) during which time, and as expected, the vehicle speed decreased at an almost constant rate (see speed profile in Figure 27). For the analysis and algorithms developed under this project, the data used was further refined by extracting only segments of data in which the $\boldsymbol{B} \boldsymbol{A P r}$ was truly constant. For example, in the braking event shown in Figure 27, two data points consistent of the 
average $\boldsymbol{B A P r}$ and corresponding deceleration were extracted and added to the database of information that was used for the analysis and brake assessment algorithms. These two points were (23.9 psi, $0.164 \mathrm{~g})$ and (23.2 psi, $0.166 \mathrm{~g}$ ), respectively, corresponding to the highlighted segments of the speed and brake application pressure profiles presented in Figure 27.

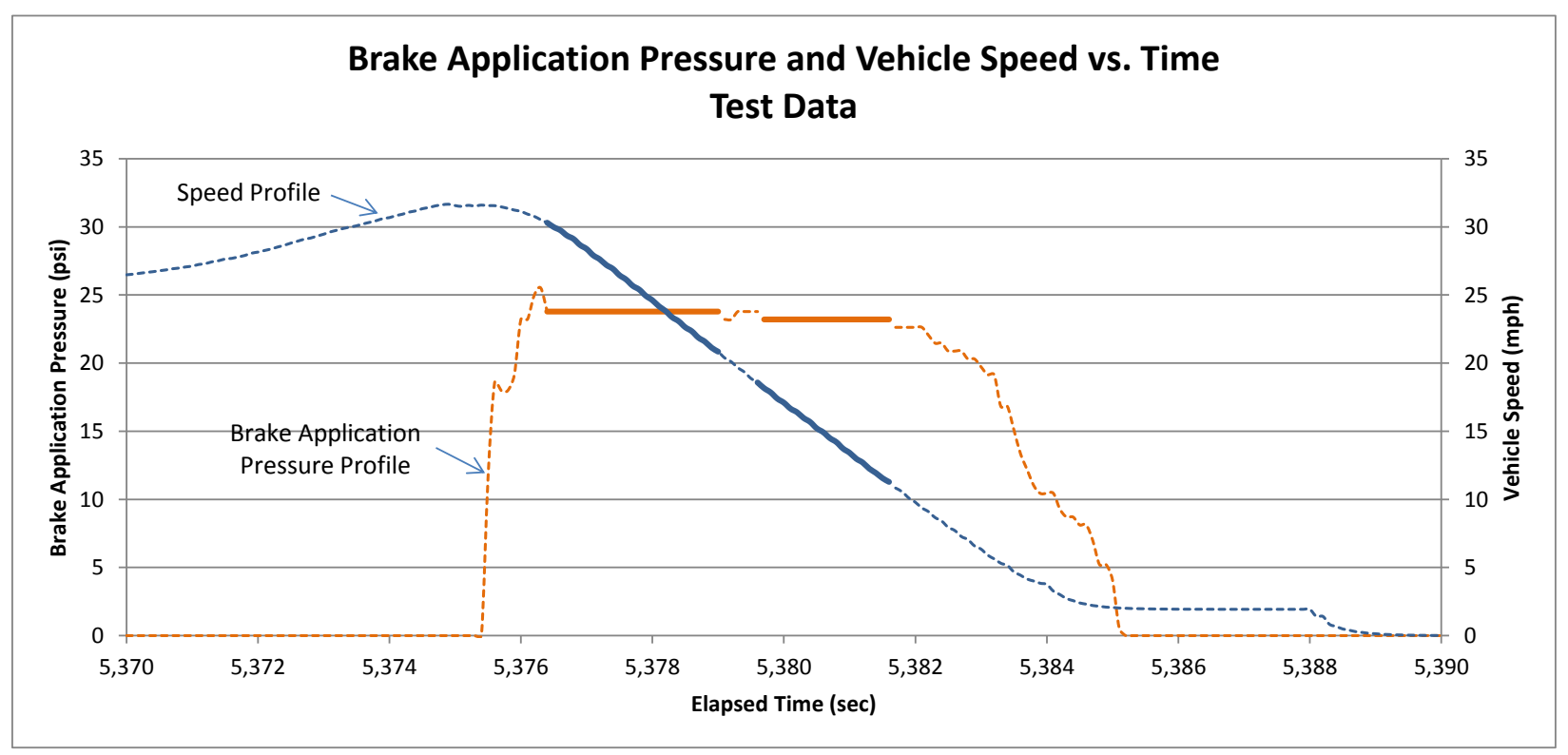

Figure 27. Brake application pressure and vehicle speed vs. time (test data segment).

In addition to $\boldsymbol{B A P r}$ and deceleration information, the algorithms that processed the data also extracted other relevant information such as vehicle weight (as provided by the weight sensors deployed on the steer and drive axles), $\boldsymbol{B A P r}$ variability (a measure of "how constant" the brake application pressure was for the data segment considered), and roadway grade (an important variable that has a direct effect on the vehicle deceleration that can be achieved at a certain $\boldsymbol{B A P r}$ ). The vehicle weight was computed as the average weight of the measurements provided by the sensors for the interval considered (i.e., the interval for which the $\boldsymbol{B A P r}$ was constant). The $\boldsymbol{B A P r}$ variability was calculated as the ratio of the standard deviation of the pressure for the interval considered divided by the average pressure for that interval. A low value for this variable would be an indication that the brake application pressure was constant ${ }^{1}$. The roadway grade was computed by using the altitude provided by the on-board GPS device. For the segment under consideration, a line was fitted using the altitude readings. The slope of that line, in percentage, was assigned as the roadway grade for that particular observation. Figure 28 presents the speed and terrain profiles as a function of the distance traveled for the braking event discussed earlier. For the two data segments presented above, the roadway grades were $-0.7 \%$ and $-1.3 \%$, respectively.

\footnotetext{
${ }^{1}$ This variable depends on the level (i.e., average value) of the $\boldsymbol{B A P r}$. However, and as discussed in sections 1 and 2 of this report, under normal operations the brake application pressure was typically between 10 and 30 psi, so variations in the average value $\boldsymbol{B} \boldsymbol{A P r}$ did not introduce a big error in the calculations of the $\boldsymbol{B A P r}$ variability. In any case, the $\boldsymbol{B A P r}$ variability was used as a screening tool to discard information in which $\boldsymbol{B A P r}$ was not constant "enough."
} 


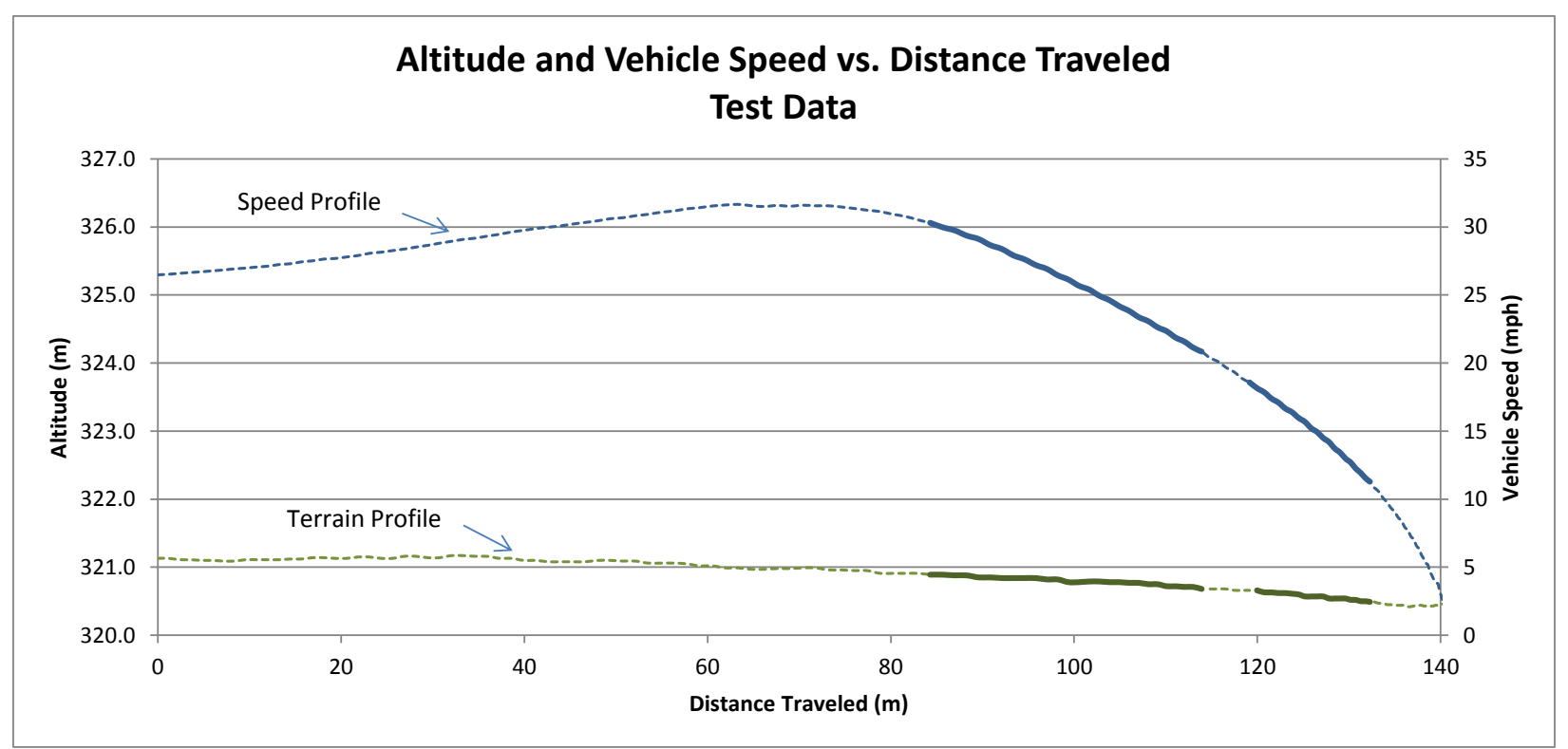

Figure 28. Altitude and vehicle speed vs. distance traveled (test data segment).

All the data collected during the tests was processed in the same way as described above. Table 2 and Table 3 present summary statistics of the processed data for medium- and heavy-load conditions tested, respectively. In these and subsequent tables, the column labeled "Length" presents statistics related to the length of the intervals for which the average $\boldsymbol{B A P r}$ and decelerations were calculated. Notice that any interval that was less than 1 second was not included in the database. Considering only cases with very low $\boldsymbol{B A P r}$ variability and intervals equal to or larger than 1 second, there were 24 observations for the medium load condition (including the two observations presented in Figure 27 and Figure 28), and 45 observations for the heavy-load case.

Table 2. Test Statistics - Medium Load

\begin{tabular}{lrcrrrr}
\hline & $\begin{array}{c}\text { Length } \\
(\mathbf{s})\end{array}$ & $\begin{array}{c}\boldsymbol{B A P r} \\
\text { Variability }\end{array}$ & $\begin{array}{c}\boldsymbol{B A P r} \\
(\mathbf{p s i})\end{array}$ & $\begin{array}{c}\text { Dec. } \\
(\mathbf{g})\end{array}$ & $\begin{array}{c}\text { Weight } \\
(\mathbf{l b s})\end{array}$ & $\begin{array}{c}\text { Grade } \\
(\boldsymbol{\%})\end{array}$ \\
\hline Count & 24 & 24 & 24 & 24 & 24 & 24 \\
Mean & 2.34 & 0.0076 & 21.95 & 0.16 & 27,175 & -1.22 \\
Standard. Deviation & 1.73 & 0.0038 & 5.89 & 0.05 & 422 & 1.52 \\
Maximum & 7.10 & 0.0158 & 32.51 & 0.25 & 27,857 & 1.84 \\
Minimum & 1.00 & 0.0031 & 10.41 & 0.06 & 26,209 & -4.63 \\
\hline
\end{tabular}

Table 3. Test Statistics - Heavy Load

\begin{tabular}{lccrrrr}
\hline & $\begin{array}{c}\text { Length } \\
(\mathbf{s})\end{array}$ & $\begin{array}{c}\boldsymbol{B} \boldsymbol{B A P r} \\
\text { Variability }\end{array}$ & \multicolumn{1}{c}{$\begin{array}{c}\boldsymbol{B} \boldsymbol{c} \text { (psi) } \\
\text { (psi) }\end{array}$} & $\begin{array}{c}\text { Dec. } \\
(\mathbf{g})\end{array}$ & $\begin{array}{c}\text { Weight } \\
(\text { lbs })\end{array}$ & $\begin{array}{c}\text { Grade } \\
(\boldsymbol{\%})\end{array}$ \\
\hline Count & 45 & 45 & 45 & 45 & 45 & 45 \\
Mean & 2.07 & 0.0073 & 22.22 & 0.14 & 32,617 & -0.80 \\
Standard Deviation & 0.91 & 0.0036 & 5.62 & 0.04 & 452 & 1.15 \\
Maximum & 5.90 & 0.0191 & 34.41 & 0.22 & 33,465 & 2.74 \\
Minimum & 1.00 & 0.0037 & 12.72 & 0.06 & 31,694 & -3.47 \\
\hline
\end{tabular}


For the two loading conditions, the vehicle was weighed at the Greene County, Tennessee Inspection Station. The information collected at the scale is presented in Table 4.

Table 4. Test Vehicle Weights

\begin{tabular}{lcc}
\hline & $\begin{array}{c}\text { Medium Load } \\
(\mathbf{l b s})\end{array}$ & $\begin{array}{c}\text { Heavy Load } \\
(\mathbf{l b s})\end{array}$ \\
\hline Steer Axle & 9,180 & 9,390 \\
Drive Axle & 14,560 & 20,480 \\
Trailer Axle & 9,150 & 14,150 \\
\hline Total $(\mathbf{G V W})$ & $\mathbf{3 2 , 8 9 0}$ & $\mathbf{4 4 , 0 2 0}$ \\
\hline
\end{tabular}

The field data was also processed using the same methodology applied to the information collected during the tests. Figure 29 show two data points collected while the vehicle was under normal operations.

Although the brake application pressure profile is not as well "shaped" as those of the braking events of the controlled experiments (see Figure 27), the applied methodology can still find segments of constant $\boldsymbol{B A P r}$ (see Figure 29). Figure 30 shows the same two data segments on the speed and terrain profiles. Notice that in the real-world data the roadway grades can be (and in some cases were) much larger than those corresponding to the data collected during the tests conducted at the inspection station.

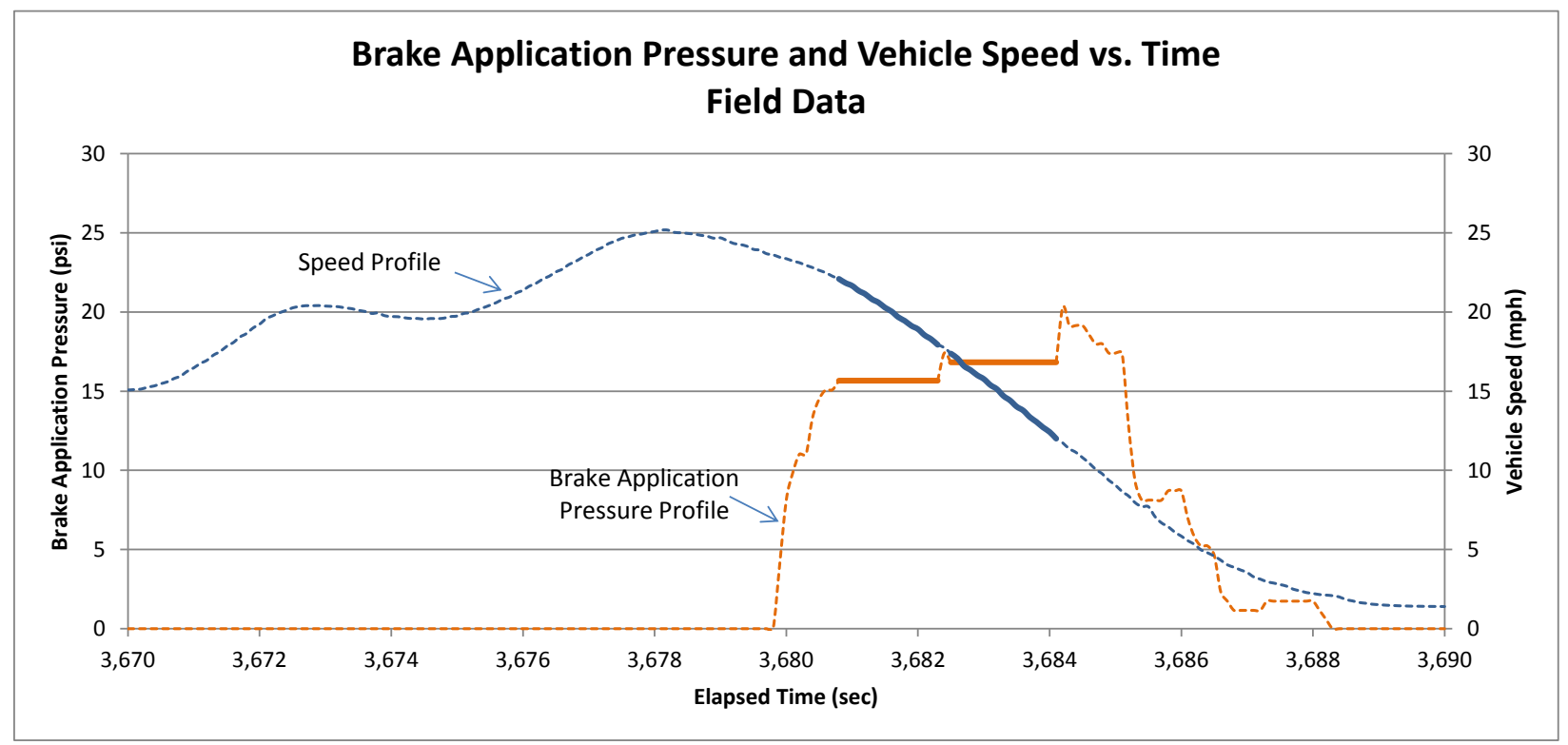

Figure 29. Brake application pressure and vehicle speed vs. time (field data segment). 


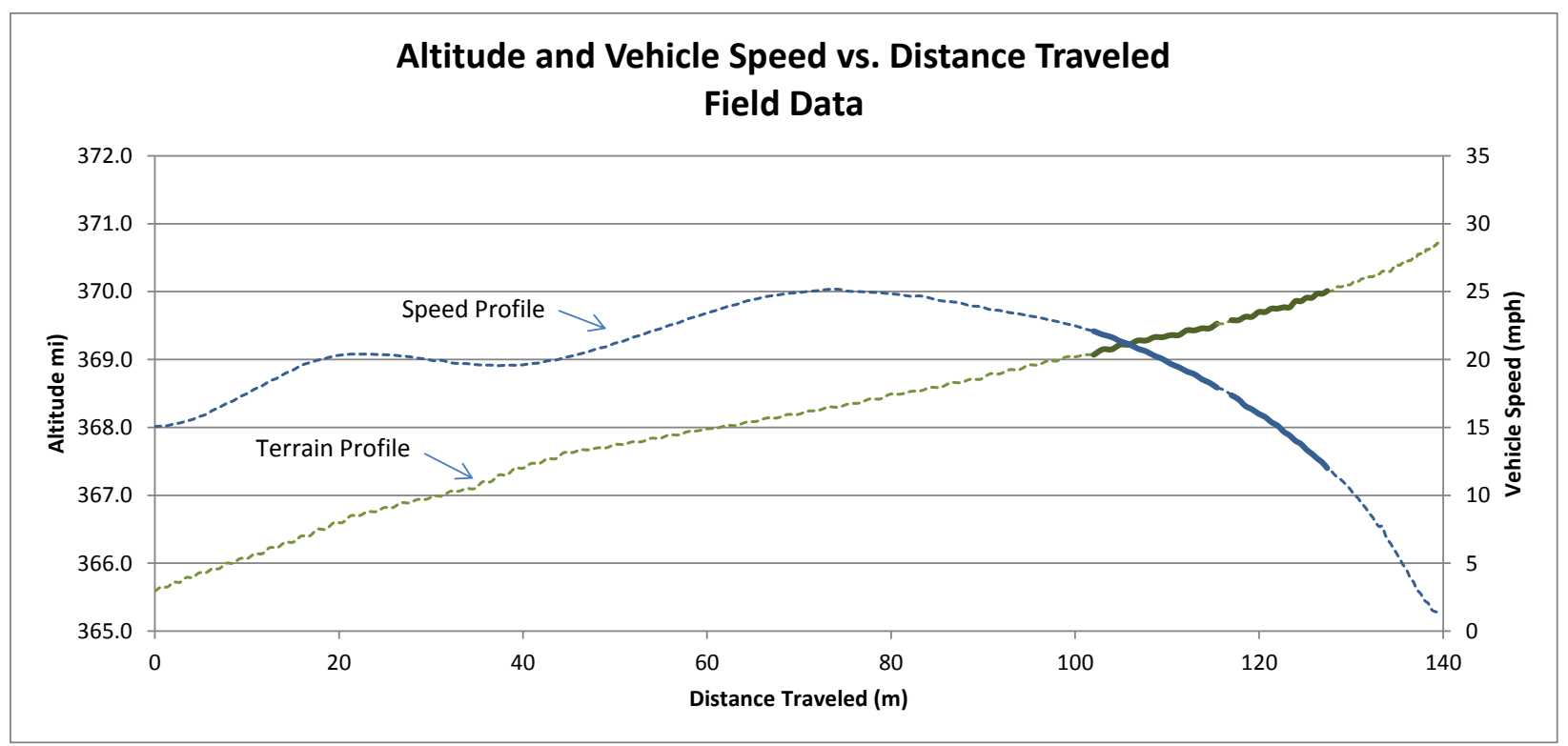

Figure 30. Altitude and vehicle speed vs. distance traveled (field data segment).

Table 5 presents summary statistics of the processed field data for medium-load condition. As it was the case with the test data, any interval that was less than 1 second was not included in the database of fieldcollected information. Considering only cases with very low $\boldsymbol{B A P r}$ variability and intervals equal to or larger than 1 second, there were 15 observations for the medium load condition (including the two observations presented in Figure 29 and Figure 30).

Table 5. Field Data Statistics - Medium Load

\begin{tabular}{lccrrrr}
\hline & $\begin{array}{c}\text { Length } \\
(\mathbf{s})\end{array}$ & $\begin{array}{c}\boldsymbol{B A P r} \\
\text { Variability }\end{array}$ & \multicolumn{1}{c}{$\begin{array}{c}\boldsymbol{B A P r} \\
(\mathbf{p s i})\end{array}$} & \multicolumn{1}{c}{$\begin{array}{c}\text { Dec. } \\
(\mathbf{g})\end{array}$} & \multicolumn{1}{c}{$\begin{array}{c}\text { Weight } \\
(\mathbf{l b s})\end{array}$} & \multicolumn{1}{c}{$\begin{array}{c}\text { Grade } \\
(\boldsymbol{\%})\end{array}$} \\
\hline Count & 15 & 15 & 15 & 15 & 15 & 15 \\
Mean & 1.53 & 0.0131 & 16.75 & 0.12 & 26,701 & -0.70 \\
Standard Deviation & 0.87 & 0.0118 & 3.79 & 0.04 & 498 & 3.72 \\
Maximum & 4.50 & 0.0528 & 26.15 & 0.20 & 27,728 & 5.74 \\
Minimum & 1.00 & 0.0046 & 10.97 & 0.04 & 26,121 & -8.88 \\
\hline
\end{tabular}

\subsection{COMPARISON OF REAL-WORLD AND SCRIPTED STOPPING DATA}

The first step in developing a system that can assess the condition of a vehicle's brakes in real-time is to determine if the data collected in the field (i.e., day-to-day operations) provides the same information as the one obtained from controlled tests. Figure 31 presents the data summarized in Table 2 and Table 5 in graphical form. For each dataset, test and field, the figure also shows the regression line that best fits the collected data. 


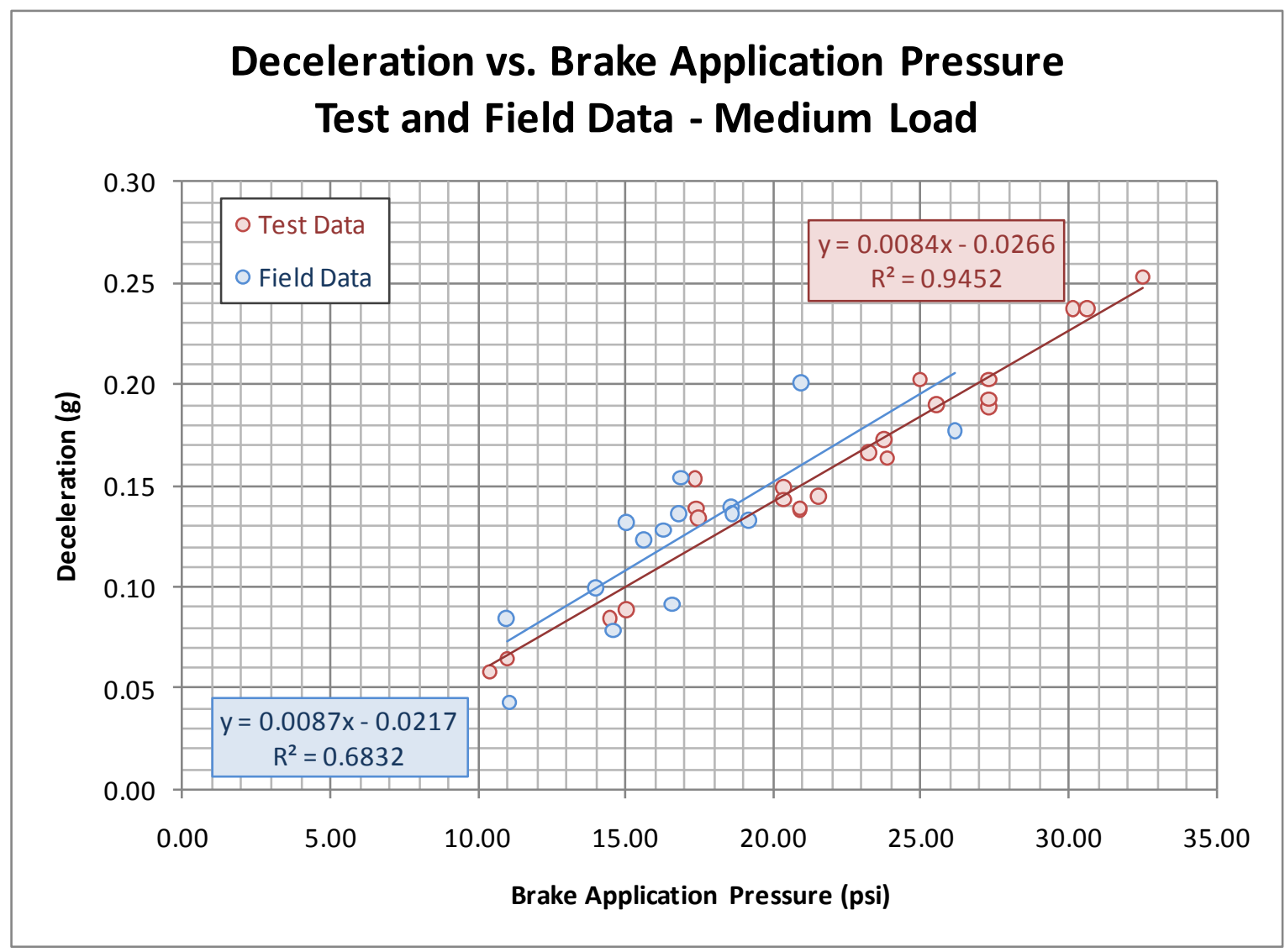

Figure 31. Deceleration vs. brake application pressure - test and field data - medium load.

In the case under consideration, the response variable (i.e., deceleration $\boldsymbol{d}$ ) and the explanatory variable (i.e., brake application pressure $\boldsymbol{B A P r}$ ) are measured for two distinct groups (i.e., test and field data). In order to compare the two regression lines, we tested the hypothesis of coincidence, that is, the two relationships are exactly the same (i.e., both test and field data describe the same phenomenon). In order to test the hypotheses of coincidence (i.e., parallelism and equality of intercepts of the two lines), we built a statistical model which describes the relationships between the response variable $\boldsymbol{d}$ and the explanatory variable $\boldsymbol{B A P r}$, for the two groups, indexed by a dummy variable $\boldsymbol{t}$. We built the following model:

$$
d_{i}=\beta_{0}+\beta_{1} B A P r_{i}+\beta_{2} t_{i}+\beta_{3} B A P r_{i} \times t_{i}+\varepsilon_{i} \quad(\text { Eq. 1) }
$$

with $\varepsilon_{i}$ denoting independent, identically normally-distributed error terms ${ }^{2}$. The dummy variable $\boldsymbol{t}$ takes the value of 1 for observations obtained from the tests, and 0 otherwise (i.e., field observations).

Therefore, the model presented in (Eq. 1 provides models for each of the two groups by letting $t$ take the value 1 or 0 , respectively:

$$
\begin{aligned}
& \text { Group } \left.1 \text { Test Data }(t=1): d=\left(\beta_{0}+\beta_{2}\right)+\left(\beta_{1}+\beta_{3}\right) B A P r+\varepsilon \quad \text { (Eq. } 2\right) \\
& \text { Group } 2 \text { Field Data }(t=0): d=\beta_{0}+\beta_{1} B A P r+\varepsilon \quad \text { (Eq. 3) }
\end{aligned}
$$

\footnotetext{
${ }^{2}$ We assume that the variances are equal in the two groups. The estimators of the variances - i.e., the square of the standard deviations of the variable $\boldsymbol{d}$ - as shown in Table 2 and Table 5 are very close, so this assumption is valid.
} 
The hypothesis of coincidence is the hypothesis that for the models presented in (Eq. 2 and (Eq. 3 the slope parameters are the same and the intercept parameters are also the same for the two groups. That is, it is the hypothesis

$$
\left.H_{0}: \beta_{2}=\beta_{3}=0 \quad \text { (Eq. } 4\right)
$$

If $H_{0}$ is true, the model presented in (Eq. 3 applies to both groups (test and field data).

In order to test the null hypothesis presented in (Eq. 4 we used the analysis of variance (ANOVA) tables for the data which are shown in Table 6 and Table 7 (note: in these and subsequent tables d.f. stands for degrees of freedom; F-value is the test statistic computed as the ratio of the mean square of the model and the mean square of the error; and the $\mathrm{p}$-value is the $\mathrm{F}$ probability distribution value corresponding to the F-value).

Table 6. ANOVA Table A (No Weight Adjustments)

\begin{tabular}{lrcccc}
\hline Source & \multicolumn{1}{c}{ d.f. } & $\begin{array}{c}\text { Sum of } \\
\text { Squares }\end{array}$ & $\begin{array}{c}\text { Mean } \\
\text { Square }\end{array}$ & F-value & p-value \\
\hline Model & 3 & 0.08342 & 0.02781 & 94.1340 & $<0.00001$ \\
Error & 35 & 0.01034 & 0.00030 & & \\
\hline Total & 38 & 0.09376 & & & \\
\hline
\end{tabular}

Table 7. ANOVA Table B (No Weight Adjustments)

\begin{tabular}{lcc}
\hline Source & d.f. & $\begin{array}{c}\text { Sequential Sum } \\
\text { of Squares }\end{array}$ \\
\hline $\boldsymbol{B A P r}$ & 1 & 0.08278 \\
$\boldsymbol{T}$ & 1 & 0.00063 \\
$\boldsymbol{B} \boldsymbol{A P r * t}$ & 1 & 0.00001 \\
\hline
\end{tabular}

With the information contained in these two tables the statistics $\boldsymbol{F}$ can be computed as

$$
F=\frac{(0.00063+0.00001) / 2}{0.00030}=1.0897 \quad(\text { Eq. 5) }
$$

Comparing with an $\boldsymbol{F}(2,35)$-distribution, we get $\boldsymbol{F}(2,35)=0.3475<1.0897$, which indicates that the null hypothesis $H_{0}(($ Eq. 4$)$ is rejected, and therefore the two lines are not coincident.

A visual inspection of the data (see Figure 31) indicates that while the two regression lines are parallel (i.e., they have almost the same slope), they have different intercepts. Moreover, the regression line corresponding to the field data is above the test data line. As described in Section 3.1.3, this could be an indication that the vehicle weight was lighter for the data labeled as medium-load for the field information considered in the analysis. Notice that the vehicle weight is obtained from the Air-Weigh sensors, which determines the drive-axle weight by using air-bag pressure information. These readings, therefore, are sensitive to the vehicle acceleration and deceleration. That is, when the vehicle is decelerating (or accelerating), the drive-axles air bags would be subject to higher (or lower) pressure due to the inertia of the trailer. ORNL has investigated this effect in a different project in which the same vehicle-weighing technology was used. ${ }^{3}$

\footnotetext{
${ }^{3}$ Capps G., O. Franzese, B. Knee, M. B. Lascurain, and P. Otaduy, Class- 8 Heavy Truck Duty Cycle Project Final
} 
The vehicle-weight data collected from the Air-Weigh sensors during the tests was plotted as a function of the $\boldsymbol{B A P r}$, for both medium- and heavy-load conditions. Figure 32 shows that in both cases there is a reasonably linear relationship ${ }^{4}$ between $\boldsymbol{B A P r}$ and the average weight computed for each data segment using information collected from the deployed weight sensors.

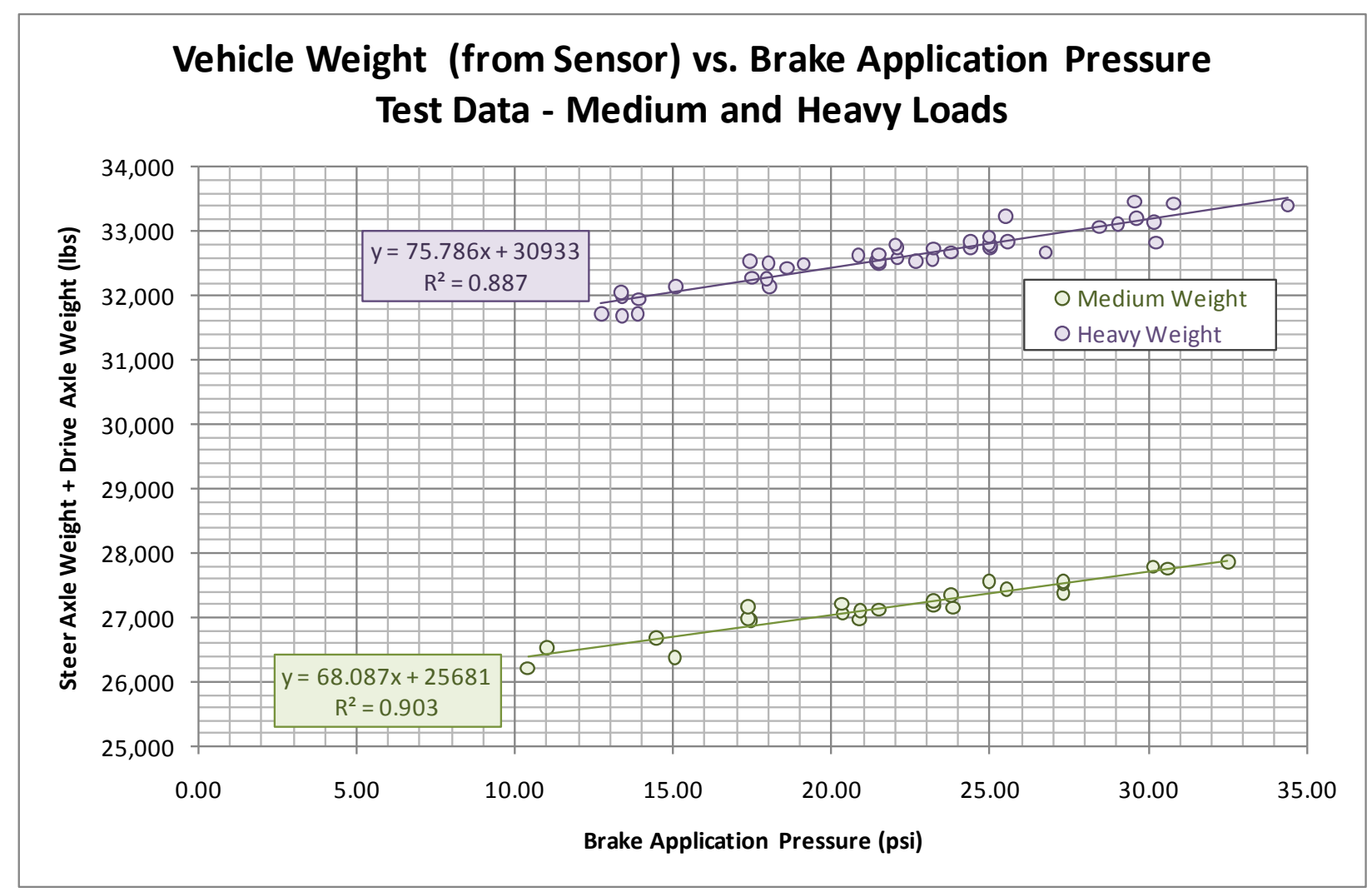

Figure 32. Vehicle weight (from on-board sensor) vs. brake application pressure - test data medium and heavy loads.

Using the information collected during the tests regarding $\boldsymbol{B A P r}$ and average weight for each data segment, as well as the vehicle's actual weight (see Table 4) it is possible to build a model that can provide vehicle weight (either steer + drive axle, $\boldsymbol{S D \boldsymbol { w }}$, loads or total vehicle weight, $\boldsymbol{T V} \boldsymbol{w}$ ) as a function of the $\boldsymbol{B A P r}$. This model(s) can then be used with the field data to make corrections to the measured weight (i.e., obtain the actual weight) so the data can be correctly compared to the test data. (Eq. 6 and (Eq. 7 present the steer + drive axles weight and total vehicle weight prediction models, respectively, as a function of the measured $\boldsymbol{B A P r}$ and steer + drive axles weights provided by the sensors (SDws). The models' coefficient of determination was very high (i.e., $\boldsymbol{R}^{2}=0.9973$ ) indicating a high linear correlation between the explanatory and dependent variables.

$$
S D w=83.29 B A P r+1.12 S D w s+4,849.3 \quad(\text { Eq. } 6)
$$

Report (ORNL/TM-2008/122), 2008.

${ }^{4}$ The inertial force generated by the trailer is proportional to the trailer weight and the coefficient of proportionality is the vehicle deceleration, which in turn is proportional to the brake application pressure. Therefore, it is expected that the relationship between $\boldsymbol{B A P r}$ and the weight measured by the sensors will be linear as well. 


$$
T V w=151.06 B A P r+2.03 S D w s+18,969.5 \quad(\text { Eq. 7) }
$$

Using the model presented in (Eq. 6, the weight corresponding to the field data summarized in Table 6 was adjusted to determine the actual weight. Figure 33 shows a graphical representation of the effect of the weight adjustment. For each data point in that figure, the abscissa (x-axis) represents the average weight obtained from the on-board weight sensors, and the ordinate (y-axis) represents the adjusted weight (predicted actual weight). The diagonal line shows the locus of the points for which the sensor and predicted weight are the same. The fact that the field data is located below that line is an indication that, as expected, the sensors overestimate weight during vehicle deceleration events. Table 8 presents the same information shown in Table 5, but this time with the addition of information related to the adjusted weight computed using (Eq. 6. Comparing the "Weight" and "Adj. Weight" information, the data that was determined to be "medium-load" data by using the weight provided by the sensors, was on average 3,000 lbs lighter (steer + drive axles loads) than what the sensors indicated for the brake events considered.

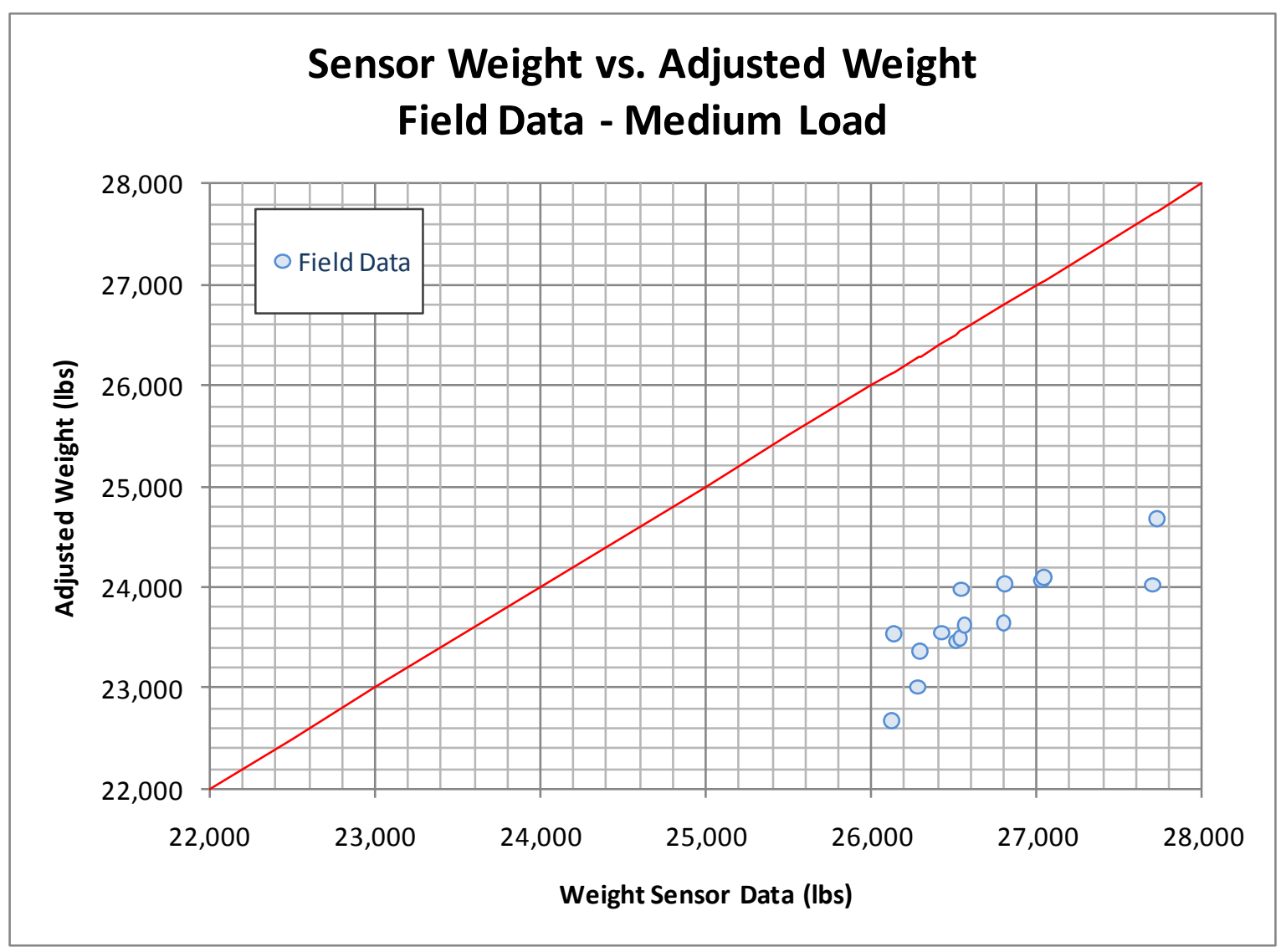

Figure 33. Sensor weight vs. adjusted weight - field data - medium load. 
Table 8. Field Data Statistics -Medium Load - Original and Adjusted Weight

\begin{tabular}{lrrrrrrr}
\hline & $\begin{array}{c}\text { Length } \\
(\mathbf{s})\end{array}$ & $\begin{array}{c}\boldsymbol{B} \boldsymbol{B A P r} \\
\text { Variability }\end{array}$ & $\begin{array}{c}\boldsymbol{B} \boldsymbol{A P r} \\
(\mathbf{p s i})\end{array}$ & $\begin{array}{c}\text { Dec. } \\
(\mathbf{g})\end{array}$ & $\begin{array}{c}\text { Weight } \\
(\mathbf{l b s})\end{array}$ & $\begin{array}{c}\text { Adj. } \\
\text { Weight }\end{array}$ & $\begin{array}{c}\text { Grade } \\
(\%)\end{array}$ \\
\hline Count & 15 & 15 & 15 & 15 & 15 & 15 & 15 \\
Mean & 1.53 & 0.0131 & 16.75 & 0.12 & 26,701 & 23,683 & -0.70 \\
Standard Deviation & 0.87 & 0.0118 & 3.79 & 0.04 & 498 & 487 & 3.72 \\
Maximum & 4.50 & 0.0528 & 26.15 & 0.20 & 27,728 & 24,681 & 5.74 \\
Minimum & 1.00 & 0.0046 & 10.97 & 0.04 & 26,121 & 22,684 & -8.88 \\
\hline
\end{tabular}

The weight data for all the field-collected information was adjusted using the model presented in (Eq. 6. After that, the information was screened using information about $\boldsymbol{B A P r}$ variability, and only those data points with low variability were selected for further consideration. Out of this screened data set, the data points $(\boldsymbol{B A P r}, \boldsymbol{d})$ that had associated adjusted weights that were within the range of the actual mediumload as defined in the tests (i.e., 23,760 lbs for the steer and drive axle loads) were selected. The resulting 24 field-collected points are plotted in Figure 34 together with the test data. The field test information is also summarized in Table 9. When the weight was adjusted for the test medium-load information, the average weight was $23,781 \mathrm{lbs}$ (compared to $23,760 \mathrm{lbs}$ measured at the scale [Table 4], and with $23,683 \mathrm{lbs}$ for the field data [Table 8]).

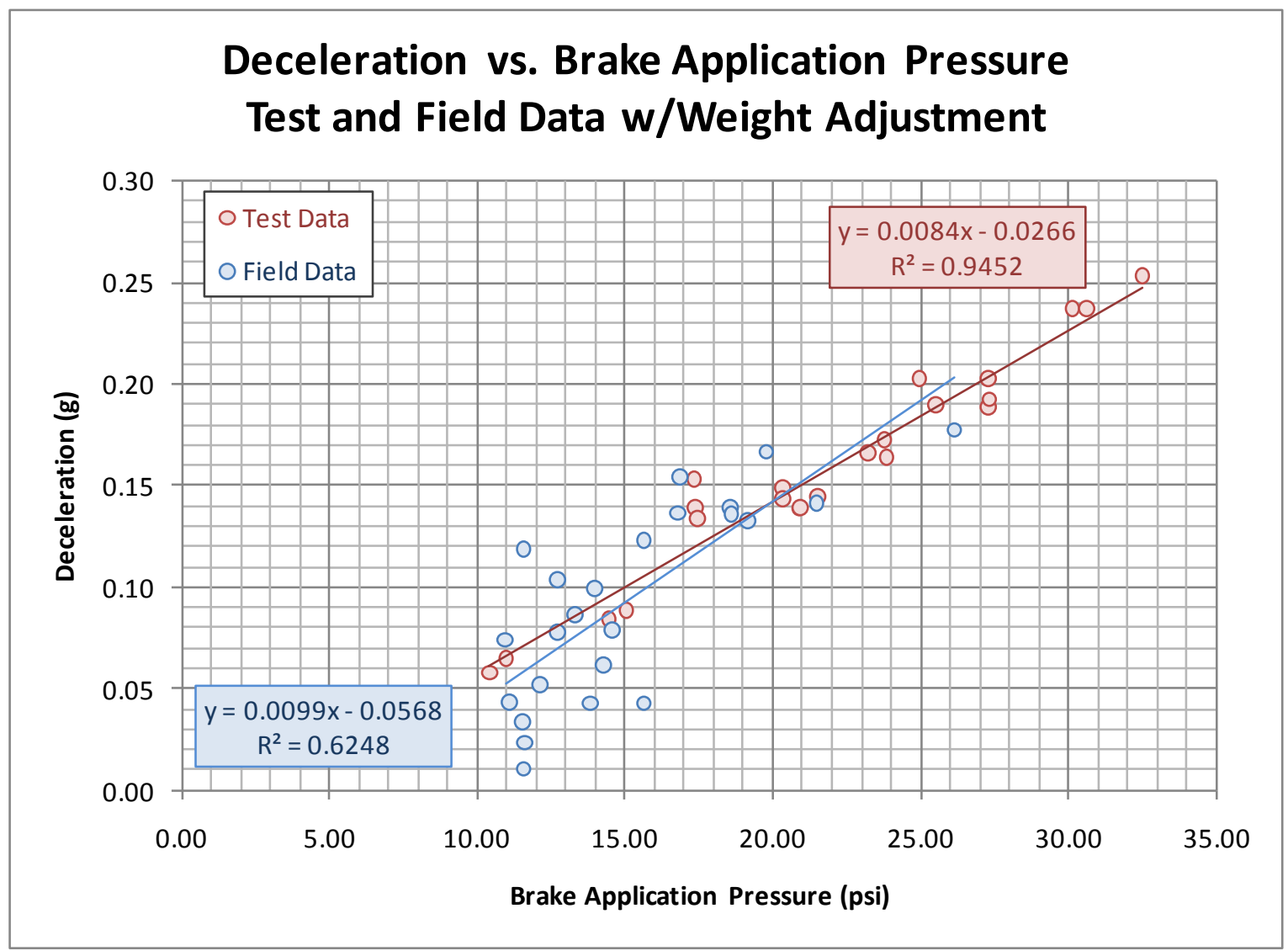

Figure 34. Deceleration vs. brake application pressure - test and field data with weight adjustment - medium load. 
Table 9. Field Data Statistics - Medium Load - Adjusted Weight

\begin{tabular}{lrrrrrr}
\hline & $\begin{array}{c}\text { Length } \\
(\mathbf{s})\end{array}$ & $\begin{array}{c}\boldsymbol{B} \text { BPr } \\
\text { Variability }\end{array}$ & $\begin{array}{c}\text { BAPr } \\
(\mathbf{p s i})\end{array}$ & \multicolumn{1}{c}{$\begin{array}{c}\text { Dec. } \\
(\mathbf{g})\end{array}$} & $\begin{array}{c}\text { Adj. } \\
\text { Weight } \\
(\mathbf{l b s})\end{array}$ & $\begin{array}{c}\text { Grade } \\
(\boldsymbol{\%})\end{array}$ \\
\hline Count & 24 & 24 & 24 & 24 & 24 & 24 \\
\multicolumn{1}{c}{ Mean } & 2.07 & 0.0155 & 15.19 & 0.09 & 23,189 & -2.08 \\
Standard Deviation & 1.06 & 0.0142 & 3.86 & 0.05 & 792 & 4.13 \\
Maximum & 5.30 & 0.0712 & 26.15 & 0.18 & 24,681 & 6.28 \\
Minimum & 1.20 & 0.0046 & 10.97 & 0.01 & 22,055 & -10.73 \\
\hline
\end{tabular}

A visual inspection of Figure 34 clearly indicates that the slopes of the regression lines for the test and field weight-corrected data are not the same. The visual inspection also shows that there are several observations very close to those observed with the crack pressure (approximately $10 \mathrm{psi}$ ) that have very low associated deceleration values. Those four data points were eliminated from further consideration. The remaining 20 observations, summarized in Table 10 and graphed in Figure 35, were used for the statistical tests.

Table 10. Field Data Statistics - Medium Load - Adjusted Weight - No Outliers

\begin{tabular}{lrrrrrr}
\hline & $\begin{array}{c}\text { Length } \\
(\mathbf{s})\end{array}$ & $\begin{array}{c}\boldsymbol{B A P r} \\
\text { Variability }\end{array}$ & $\begin{array}{c}\boldsymbol{B A P r} \\
(\mathbf{p s i})\end{array}$ & $\begin{array}{c}\text { Dec. } \\
(\mathbf{g})\end{array}$ & $\begin{array}{c}\text { Adj. } \\
\text { Weight } \\
(\mathbf{l b s})\end{array}$ & $\begin{array}{c}\text { Grade } \\
(\%)\end{array}$ \\
\hline Count & 20 & 20 & 20 & 20 & 20 & 20 \\
Mean & 2.15 & 0.0152 & 15.71 & 0.11 & 23,352 & -0.80 \\
Standard Deviation & 1.14 & 0.0148 & 3.96 & 0.04 & 734 & 3.13 \\
Maximum & 5.30 & 0.0712 & 26.15 & 0.18 & 24,681 & 6.28 \\
Minimum & 1.20 & 0.0046 & 10.97 & 0.04 & 22,057 & -5.40 \\
\hline
\end{tabular}

A new Test of Coincidence was performed using the ANOVA tables corresponding to the tests and weight-corrected field data. The information is presented in Table 11 and Table 12.

Table 11. ANOVA Table A (Weight Adjusted Data)

\begin{tabular}{lrrrrl}
\hline Source & d.f. & $\begin{array}{c}\text { Sum of } \\
\text { Squares }\end{array}$ & $\begin{array}{c}\text { Mean } \\
\text { Square }\end{array}$ & F-value & p-value \\
\hline Model & 3 & 0.10695 & 0.03565 & 98.9822 & $<0.0001$ \\
Error & 40 & 0.01441 & 0.00036 & & \\
\hline Total & 43 & 0.12135 & & & \\
\hline
\end{tabular}

Table 12. ANOVA Table B (Weight Adjusted Data)

\begin{tabular}{l|cc}
\hline Source & d.f. & $\begin{array}{c}\text { Sequential Sum } \\
\text { of Squares }\end{array}$ \\
\hline $\boldsymbol{B A P r}$ & 1 & 0.10694 \\
$\quad$ Type & 1 & 0.00001 \\
BAPr*Type & 1 & 0.00000 \\
\hline
\end{tabular}




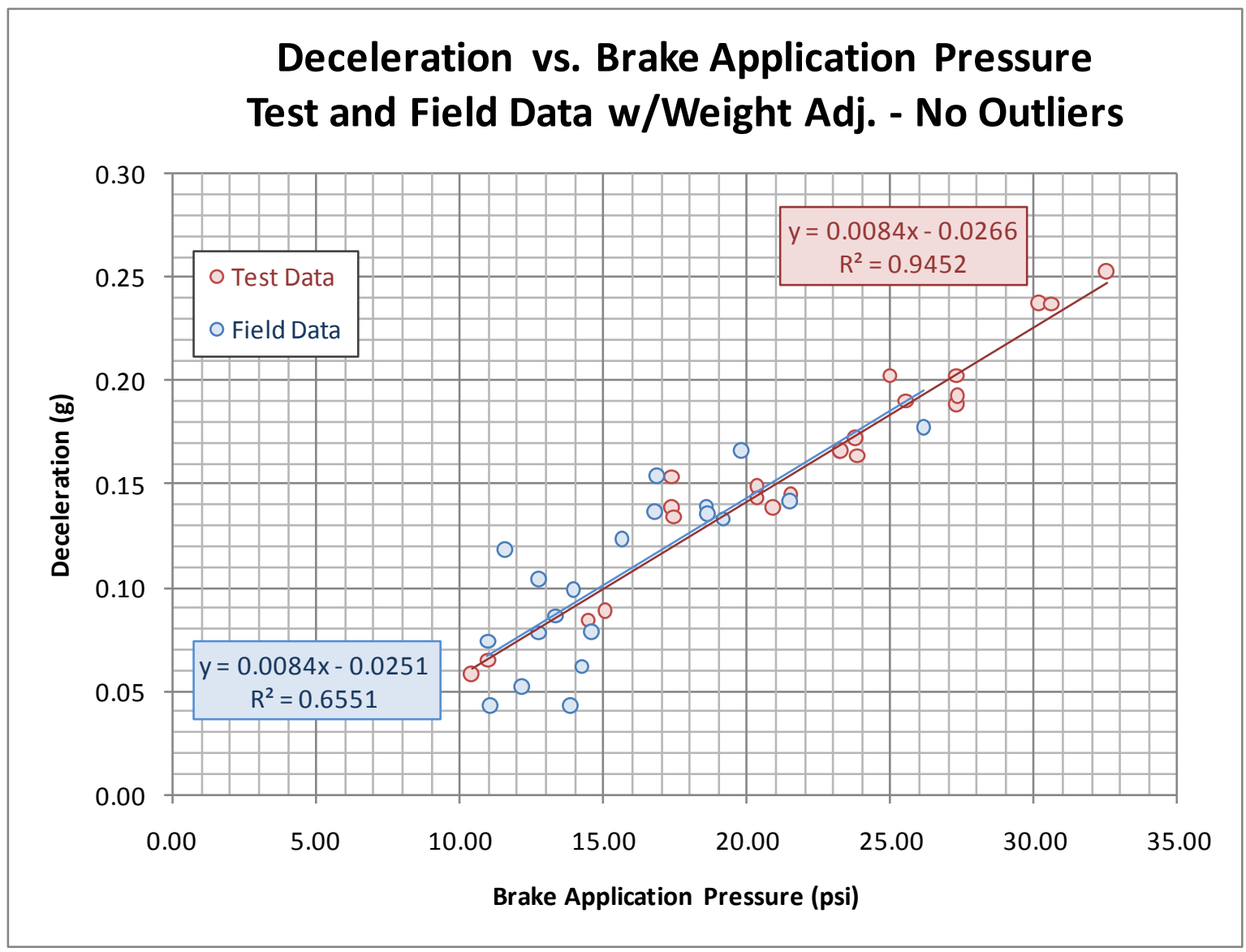

Figure 35. Deceleration vs. brake application pressure - test and field data with weight adjustment: no outliers - medium load.

With the information contained in these two tables the statistics $\boldsymbol{F}$ can be computed as

$$
F=\frac{(0.00001+0.00000) / 2}{0.00036}=0.0161 \quad(\text { Eq. } 8)
$$

Comparing with an $\boldsymbol{F}(2,40)$-distribution, we get $\boldsymbol{F}(2,40)=0.9841>0.0161$, which indicates that the null hypothesis $H_{0}$ ((Eq. 4) cannot be rejected with a high level of confidence, thus concluding that the two lines are coincident. That is, after adjusting the data to correct the weight provided by the sensors, and screening for low-deceleration values for data points in which the $\boldsymbol{B A P r}$ is very close to the crack pressure, the field data (data collected during normal operations) provides the same information as the one obtained in highly controlled experiments.

(Eq. 9 presents the final model showing the relationship between brake application pressure and deceleration for vehicles with medium-size loads (note: for the field data selected, the steer + drive axle loads varied between 22,000 lbs and 24,700 lbs; this interval contains 23,760 lbs which was the scaleweight for the medium-load tested condition).

$$
d=0.0084 B A P r-0.0249
$$


For the model presented in (Eq. 9, the coefficient of determination was $\boldsymbol{R}^{2}=0.8812$. If the vehicle weight for the medium-load case for the field data is constrained to a tighter interval (e.g., 23,200 lbs to 24,100 lbs) around the defined (i.e., tested) medium-load weight, then the relationship between BAPr and deceleration is shown in (Eq. 10, which as a coefficient of determination $\boldsymbol{R}^{2}=0.9136$. For this project, the field data was collected during a two-week period which is the reason for having so few observations that qualify as "medium-load." In a real world application, many more points would be collected and the weight intervals could be reduced to 500 or fewer pounds.

$$
d=0.0083 B A P r-0.0230 \quad \text { (Eq. 10) }
$$




\section{LESSONS LEARNED}

A number of lessons were learned from this research. First, the stopping tests were conducted in the large parking area at the Greene County Inspection Station. This provided a suitable, safe area to conduct these low-speed stopping tests without the expense of using a test track facility.

Each of the plots from the Greene County testing were generated using a single trailer (one for each day of testing). However, H.T. Hackney has a drop-and-hook operation, meaning the tractor is not consistently connected to the same trailer. As such, the set of trailer brakes involved in the real-world stops was not constant throughout the two-week testing period. In the future, all testing should be done with the same trailer as well as tractor. In real-world situations where this is not feasible such as dropand-hook operations, a longer period of data collection could be used where accurate trailer records are kept for the test period.

Using a human in the loop to conduct constant-pressure stops reduced the controllability of the data collection and consistency in applying the target pressures. However, the fact that streaming data was being collected meant that the data could be post-processed to extract precise stopping test statistics from the data (rather than use the target speeds and pressures), even though the driver could only provide approximate inputs in terms of starting speed and brake application pressure. If instrumentation could be used in future testing to perform tests more precisely, it would minimize the amount of data processing required as well as the number of repetitions needed for each test scenario. 


\section{RECOMMENDATIONS FOR FUTURE RESEARCH}

For the Phase II Pilot Test, various methods could be used to degrade brake performance for testing purposes to verify assumptions or to determine alternative ways to detect brake performance degradation. The most convenient may be to adjust stroke lengths so the pushrods are out-of-stroke. Another method might be to disable or disconnect pairs of brakes, resulting in inoperative brakes. Another methodology may be used to simulate wear or loss of friction, such as replacing brake components with their worn counterparts. Ideally, whatever method is used, a PBBT test should also be performed to quantify the performance degradation.

One area in which further tests would be useful is conducting testing at higher speeds to confirm there is indeed no relationship between deceleration and initial speed for a given brake application pressure. This was demonstrated in the 20- and 30-mph testing and is supported by the theory, but should be tested to confirm that there are no other nonlinear factors contributing to deceleration at higher speeds.

Another area in which further investigation is needed is determining how weight readings (designed for level use) are affected by sloped terrain. There is a need to determine how the grade affects the P-BF curve and whether an independent grade measurement (such as one based on GPS) is necessary. It may be possible to infer an approximate slope from other parameters such as deceleration characteristics or weight shifting.

This research focused on brake application pressures below 30 psi because previous research indicated that this was the region in which braking events typically occurred for the test vehicles. However, it is possible that other vocations see higher brake application pressures on a regular basis. As long as the typical brake application pressures lie in the linear region (up to approximately 50-60 psi), the method explored in this proof-of-concept study should be suitable for any duty cycle. Thus, the next stage of testing should also explore the middle pressure region as well.

Finally, the design of the specific test plan must include a number of safety considerations. These include restricting all testing to a closed test area, modifying brakes in a symmetrical manner, and avoiding all types of brake system alterations which are not completely reversible. 


\section{APPENDIX: LIST OF COLLECTED SIGNALS}

\begin{tabular}{|c|c|c|}
\hline No. & Signal Description & Source \\
\hline 1 & Total Vehicle Distance & J1939 \\
\hline 2 & Road Speed Limit Status & J1939 \\
\hline 3 & Wheel-Based Vehicle Speed/Road Speed & J1939 \\
\hline 4 & Front Axle Speed & J1939 \\
\hline 5 & Engine Speed & J1939 \\
\hline 6 & Current Gear & J1939 \\
\hline 7 & Selected Gear & J1939 \\
\hline 8 & Actual Gear Ratio & $\mathrm{J} 1939$ \\
\hline 9 & Output Shaft Speed & J1939 \\
\hline 10 & Transmission Selected Range & J1939 \\
\hline 11 & Transmission Current Range & J1939 \\
\hline 12 & Engine Oil Temperature & J1939 \\
\hline 13 & Intake Manifold Temperature & J1939 \\
\hline 14 & Engine Coolant Temperature & J1939 \\
\hline 15 & Boost Pressure & $\mathrm{J} 1708$ \\
\hline 16 & Fuel Rate & $\mathrm{J} 1939$ \\
\hline 17 & Instantaneous Fuel Economy & J1939 \\
\hline 18 & Actual Engine - Percent Torque & J1939 \\
\hline 19 & Percent Accelerator Pedal Position & J1939 \\
\hline 20 & Percent Load at Current Speed & J1939 \\
\hline 21 & Driver's Demand Engine - Percent Torque & J1939 \\
\hline 22 & Nominal Friction Percent Torque & J1939 \\
\hline 23 & Brake Switch & J1939 \\
\hline 24 & Clutch Switch & J1939 \\
\hline 25 & Cruise Control Accelerate Switch & J1939 \\
\hline 26 & Cruise Control Active & J1939 \\
\hline 27 & Cruise Control Coast Switch & J1939 \\
\hline 28 & Cruise Control Enable Switch & J1939 \\
\hline 29 & Cruise Control Resume Switch & $\mathrm{J} 1939$ \\
\hline 30 & Cruise Control Set Switch & J1939 \\
\hline 31 & Cruise Control Set Speed & J1939 \\
\hline 32 & Power Takeoff Governor/Status Flags & J1939 \\
\hline 33 & Power Takeoff Set Speed & J1939 \\
\hline 34 & Total Power Takeoff Hours & $\mathrm{J} 1939$ \\
\hline 35 & Battery Voltage & J1939 \\
\hline 36 & Fan Drive State & $\mathrm{J} 1939$ \\
\hline 37 & AC High Pressure Fans Switch & J1939 \\
\hline 38 & Barometric Pressure & J1939 \\
\hline
\end{tabular}




\begin{tabular}{|c|l|c|}
\hline No. & \multicolumn{1}{|c|}{ Signal Description } & Source \\
\hline 39 & Latitude & VBOX II Lite \\
\hline 40 & Longitude & VBOX II Lite \\
\hline 41 & Altitude & VBOX II Lite \\
\hline 42 & Vertical Velocity & VBOX II Lite \\
\hline 43 & Velocity over Ground & VBOX II Lite \\
\hline 44 & Longitudinal Acceleration & VBOX II Lite \\
\hline 45 & Lateral Acceleration & VBOX II Lite \\
\hline 46 & Heading & VBOX II Lite \\
\hline 47 & Satellites & VBOX II Lite \\
\hline 48 & Time UTC & VBOX II Lite \\
\hline 49 & Distance & VBOX II Lite \\
\hline 50 & Steer Axle Weight & Air-Weigh \\
\hline 51 & Drive Axle Weight & Air-Weigh \\
\hline 52 & Wiper Switch Status & Wiper Switch \\
\hline 53 & Brake Actuator Status - Left Front & E-Stroke \\
\hline 54 & Brake Actuator Status - Right Front & E-Stroke \\
\hline 55 & Brake Actuator Status - Left Rear & E-Stroke \\
\hline 56 & Brake Actuator Status - Right Rear & E-Stroke \\
\hline 57 & Lining Status - Left Front & E-Stroke \\
\hline 58 & Lining Status - Right Front & E-Stroke \\
\hline 59 & Lining Status - Left Rear & E-Stroke \\
\hline 60 & Lining Status - Right Rear & E-Stroke \\
\hline 61 & Brake Application Pressure & E-Stroke \\
\hline 62 & Tire Pressure - Left Front & Tire SafeGuard \\
\hline 63 & Tire Pressure - Right Front & Tire SafeGuard \\
\hline 64 & Tire Pressure - Left Rear Outside & Tire SafeGuard \\
\hline 65 & Tire Pressure - Left Rear Inside & Tire SafeGuard \\
\hline 66 & Tire Pressure - Right Rear Inside & Tire SafeGuard \\
\hline 67 & Tire Pressure - Right Rear Outside & Tire SafeGuard \\
\hline 68 & Tire Temperature - Left Front & Tire SafeGuard \\
\hline 69 & Tire Temperature - Right Front & Tire SafeGuard \\
\hline 70 & Tire Temperature - Left Rear Outside & Tire SafeGuard \\
\hline 71 & Tire Temperature - Left Rear Inside & Tire SafeGuard \\
\hline 72 & Tire Temperature - Right Rear Inside & Tire SafeGuard \\
\hline 73 & Tire Temperature - Right Rear Outside & Tire SafeGuard \\
\hline
\end{tabular}




\begin{tabular}{|c|c|c|c|}
\hline \multicolumn{4}{|c|}{ RTDBA POC Final Report Revision Log } \\
\hline $\begin{array}{c}\text { Revision } \\
\text { Number }\end{array}$ & Description of Change & Editor(s) & $\begin{array}{c}\text { Change Effective } \\
\text { Date }\end{array}$ \\
\hline 0 & Initial draft & MBL & 20 May 2011 \\
\hline 1 & Data analysis & OF & 13 Jun 2011 \\
\hline 2 & Complete Draft & MBL & 23 Aug 2011 \\
\hline 3 & $\begin{array}{c}\text { Incorporated internal edits; version } \\
\text { submitted to sponsor }\end{array}$ & GJC, SAM, MBL, OF & 30 Aug 2011 \\
\hline 4 & Incorporated sponsor's comments & LL,MBL & 1 Nov 2011 \\
\hline
\end{tabular}

